\title{
Comparison of 3D MT inversions for Geothermal Exploration: Case studies for Krafla and Hengill Geothermal systems in Iceland
}

1 Gudni Karl Rosenkjaer ${ }^{1,3}$, Erika Gasperikova ${ }^{2}$, Gregory A. Newman ${ }^{2}$, Knutur Arnason ${ }^{3}$, and

2 Nathaniel J. Lindsey ${ }^{2}$

$3{ }^{1)}$ University of British Columbia, 6339 Stores Road, Vancouver, BC, V6T 1Z4, Canada

$4 \quad{ }^{2)}$ Lawrence Berkeley National Laboratory, 1 Cyclotron Road, MS74R316C,

$5 \quad$ Berkeley, CA 94720

$6 \quad$ 3) Iceland Geosurvey, Grensasvegur 9, 108 Reykjavik, Iceland

$7 \quad{ }^{*}$ Corresponding author: E-mail address: grosenkj@eos.ubc.ca (G. Rosenkjaer)

9 Keywords: Magnetotellurics, 3D inversion, geothermal, Iceland, Krafla, Hengill

11 May 25, 2015

\section{Abstract}

14 The magnetotelluric (MT) method is important for exploration of geothermal systems. The

15 information on the Earth’s resistivity obtained with MT methods has been valuable in imaging

16 the hydrothermal alteration of such systems. Given its ability to recover complex resistivity

17 models for the Earth, three-dimensional (3D) MT inversion has become a common practice in 
18 geothermal exploration. However, 3D inversion is a time-consuming and complicated procedure

19 that relies on computer algorithms to search for a model that can explain the measured data to a

20 sufficient level. Furthermore, many elements of inversion require input from the practitioner,

21 which can easily bias the results. Consequently, final 3D MT results depend on various factors,

22 including the inversion code, the model mesh used to represent the Earth, data quality and

23 processing, and constraints imposed during the inversion procedure.

24 In this paper, to explore how this variability in 3D MT modeling impacts the final model, we

25 invert MT data sets from the Krafla and Hengill geothermal areas in Iceland, using three different

26 inversion codes. In each case, the modelers had the freedom to select a subset of the data and

27 implement the inversion for the respective code in an optimized way. We compare the results

28 from all the inversion codes, as well as consider the setup and assumptions made during the

29 inversion process, all of which helps enhance the robustness and quality of the results. The

30 comparison is done in multiple ways, using visual comparison of the recovered resistivity

31 models, as well as comparing the structural similarities of the models by employing a structural

32 correlation metric based on cross-gradients and other types of metrics for structural correlation.

33 This approach highlights structures that are common in all three models, and implies that these

34 structures are independent of the inversion code and necessary to fit the data.

35 All modeling results from both Krafla and Hengill are consistent to first order, recovering a

36 conductive layer on top of a resistive core typical of high temperature geothermal systems. For

37 Hengill, the models show strong structural agreement, with all inversions recovering a

38 moderately layered resistivity model but adding detail to previous work done in the area. Major

39 differences are found in areas with coarse data coverage and hence questionable model

40 resolution. Where the recovered structures in different models coincide, our confidence that these 
41 structures are well-constrained by the data is elevated, in spite of the different setup and

42 assumptions in the codes these structures are required; so they can be interpreted in terms of

43 geology with more certainty. Results from Krafla are not as consistent as results for Hengill,

44 related in part to the Krafla data being nosier than the Hengill data. The models from Krafla have

45 coinciding larger structures, but small-scale structures there are less coherent. One of the

46 consistent structures in all the models is a conductive zone reaching from a depth of $5 \mathrm{~km}$ to

47 shallower depths in the northern part of the area.

\section{$48 \quad$ 1. Introduction}

49 To investigate the subsurface using magnetotelluric (MT) measurements, we use 3D inversion,

50 which has become standard practice in geothermal exploration. MT technology enables detailed

51 imaging of the geological structures and hydrothermal alteration zones that characterize the

52 geothermal system (Árnason et al., 2010; Heise et al., 2008; Newman et al., 2008).

53 Devolvement of 3D MT inversion codes in the last decade has led to greater use of 3D inversion

54 for MT surveying. These codes formulate the inverse problem in different ways, taking advantage

55 of differing numerical algorithms to increase accuracy and accelerate the process. There has been

56 considerable effort put into researching the strengths and weaknesses of different inversion

57 approaches. Miensopust et al., (2013) reviews conclusions of two workshops hosted by the

58 Dublin Institute for Advanced Studies, wherein both code developers and practitioners/modelers

59 from academia and industry came together to assess the status of 3D modelling and inversion of

60 MT data. MT responses were calculated by the participants using the same resistivity model, and

61 responses were compared. As for inversion of MT data, synthetic data produced for mystery

62 models were inverted by the participants. An important finding was that most codes have 
63 reasonable qualitative agreement, but differences arise from how the inversion codes were set up

64 and used. Some of the factors that led to differences are pre-processing of the data; selection of

65 stations, components, and/or frequencies used for the inversion; assessment and error assignment

66 for the desired level of misfit; model discretization; and setup of the inversion constraints.

67 Geothermal systems pose additional challenges for 3D MT inversion. Geothermal systems are

68 structurally complex, often associated with volcanic complexes having significant topographic

69 relief and inhomogeneous materials. While the resistivity of geothermal systems is influenced by

70 multiple petrophysical and geological processes that have occurred in the system, hydrothermal

71 alteration, temperature, and conduction mechanisms are considered to be the major influences

72 (Flóvenz et al., 2012).

73 The goal of this work is to compare results from two geothermal fields in Iceland, Krafla and

74 Hengill, using three different 3D MT inversion codes to recover the resistivity structures of these

75 fields. The codes used in this paper are the MT3Dinv (Farquharson et al., 2002), EMGeo

76 (Newman and Alumbaugh, 2000) and WSINV3DMT (Siripunvaraporn et al., 2005). In order to

77 better assess the robustness of running an inversion on real data sets, the only restriction imposed

78 is that all the data used for the inversions be from the same pre-processed MT dataset. Otherwise,

79 the modeler had full control over how the inversion was designed (including selecting a subset

80 from the data set), both in terms of stations and frequencies, and in assessing and assigning

81 appropriate measurement uncertainties. To achieve a better control in comparing structures in the

82 models, we calculated gradients and Laplacians of the logarithmic transforms for the resulting

83 resistivity models (in addition to discussing the resulting models). We also employed cross-

84 gradients to map the structural similarities and dissimilarities between the respective models. 
The MT method is a passive electromagnetic (EM) method, in which the source fields are a wide

87 spectrum of EM waves that naturally occur due to lightning discharges and interactions between

88 the solar wind and the Earth’s magnetosphere (Cagniard, 1953). The source-wave fields are

89 considered to be arbitrarily polarized plane waves that propagate vertically into the Earth since

90 refraction of EM waves at the interface of air and Earth is high. The depth of investigation

$91\left(\delta_{M T}\right)$ can be estimated from the skin depth rule:

$92 \delta_{M T}(f)=\sqrt{\frac{\rho}{\mu \pi f}} \approx 500 \sqrt{\frac{\rho}{f}}$ in $[m]$.

93 Here $\delta_{M T}$ is an estimate of the depth at which the source wave amplitude has attenuated to $\boldsymbol{e}^{\mathbf{- 1}}$

94 from its surface value for a given frequency $(f)$ and an estimated resistivity $(\rho)$ of the subsurface.

95 By measuring orthogonal electric (E) and magnetic $(\mathrm{H})$ fields at the surface one can retrieve

96 information about the subsurface resistivity structure, from a few meters to hundreds of

97 kilometers depth. The orthogonal horizontal components of the E and $\mathrm{H}$ fields (Ex, Ey, Hx, Hy)

98 are interrelated by

$99 \quad\left[\begin{array}{l}E_{x} \\ E_{y}\end{array}\right]=\left[\begin{array}{ll}Z_{x x} & Z_{x y} \\ Z_{y x} & Z_{y y}\end{array}\right]\left[\begin{array}{l}H_{x} \\ H_{y}\end{array}\right]$,

where the surface impedance $(\mathrm{Z})$ is a $2 \times 2$ complex tensor. During recording, $\mathrm{Z}$ is calculated for each MT station as a function of frequency. By taking the ratio of the fields measured at the same

103 time, the source effects of the MT fields cancel; therefore, $\mathrm{Z}$ can be used to deduce the electrical

104 properties of the Earth, without the need for a more complete description of the MT source field

105 effects (i.e., vertically incident plane wave excitation will suffice). Impedance data are often

106 presented as apparent resistivity and phase 
$107 \quad \rho_{i j}^{a p p}(f)=\frac{1}{2 \pi \mu_{0} f}\left|Z_{i j}(f)\right|^{2} \quad$ and $\quad \phi_{i j}^{a p p}(f)=\arctan \left(\frac{\operatorname{Im}\left(Z_{i j}(f)\right)}{\operatorname{Re}\left(Z_{i j}(f)\right)}\right)$ where $i, j=\{x, y\}(3)$

108 in order to provide more intuitive values to inspect and interpret the MT response (Vozoff, 1991).

109 In this paper, as commonly done in geothermal exploration, only the off-diagonal elements are

110 used for the analysis. Using the full impedance tensor has been shown to improve recovery of

111 deep structures (e.g., Kiyan et al., 2013) and should be considered in future work. However,

112 using the full tensor demands more computational power in solving the inverse problem,

113 particularly for inverse algorithms based upon a data space implementation, and requires an

114 accurate and delicate assessment of error weights with respect to the data objective function. If

115 that requirement is not fulfilled, the inversion results may not be geologically meaningful,

116 compared to those obtained by exclusively using off-diagonal elements. Moreover, for most of

117 the data considered in this study, the on-diagonal elements of the impedance tensor are an order

118 of magnitude smaller than the off-diagonal elements, suggesting that most of the information is

119 contained in the off-diagonal elements. Test inversions were performed using the full impedance

120 and off-diagonal tensor data to assess the contribution of the on-diagonal data. These tests

121 revealed similar resistivity structures in both cases, supporting the notion that the resistivity can

122 be resolved essentially from off-diagonal tensor data.

123 Galvanic distortion, commonly referred to as static shift, is caused by near-surface

124 inhomogeneous resistivity distributions as well as topography and can create static (amplitude)

125 shifts in MT soundings. The static shift can be addressed using TEM data as described in

126 Árnason et al. (2010). Stark et al., (2013) shows how including a detailed elevation model in a

127 forward modeling of a halfspace produces considerable static shift and suggest that large part of

128 distortion can be explained by topographic affect alone. Therefore, whereas including detailed

129 topographic information into the model is beneficial, in practice it can be an expensive and 
130 cumbersome procedure with a large number of cells needed to capture the details of the

131 topography. At both Krafla and Hengill, auxiliary TEM data sets have been measured in close

132 proximity to the MT soundings, enabling a static shift correction. These TEM data have been

133 used to correct MT data for 3D inversions in Hengill (Árnason et al., 2010; Rosenkjær, 2011) and

134 for 1D inversion in Krafla (Friðleifsson et al., 2014). Rosenkjær (2011) discusses the use of

135 TEM-static-shift-corrected data, both for a synthetic geothermal system with near surface

136 resistivity inhomogenities and for MT data from the Hengill area. Rosenkjaer shows that the

137 static shift correction with TEM data recovers a moderately more agreeable resistivity model in

138 both cases compared to the inversions without the static shift correction. One of the inversion

139 codes used in this study (WSINV3DMT) does not allow for topography to be included in the

140 inversion procedure. Considering all of these factors, we decided to use the TEM static-shift-

141 corrected data for all the inversions, even though two of the inversion codes will explicitly

142 consider topographic variations.

\section{$144 \quad 2.1$ Krafla MT data}

145 The Krafla geothermal area is located in the Northern Volcanic Zone in Iceland. The area is in an

146 active rift zone with a dominant SSW-NNE fault and fissure direction, crosscut by a WNW-ESE-

147 oriented transform graben (Hjartardóttir et al., 2012). For more detailed discussion of the

148 geology, see Gasperikova et al. (2015) (this issue). 


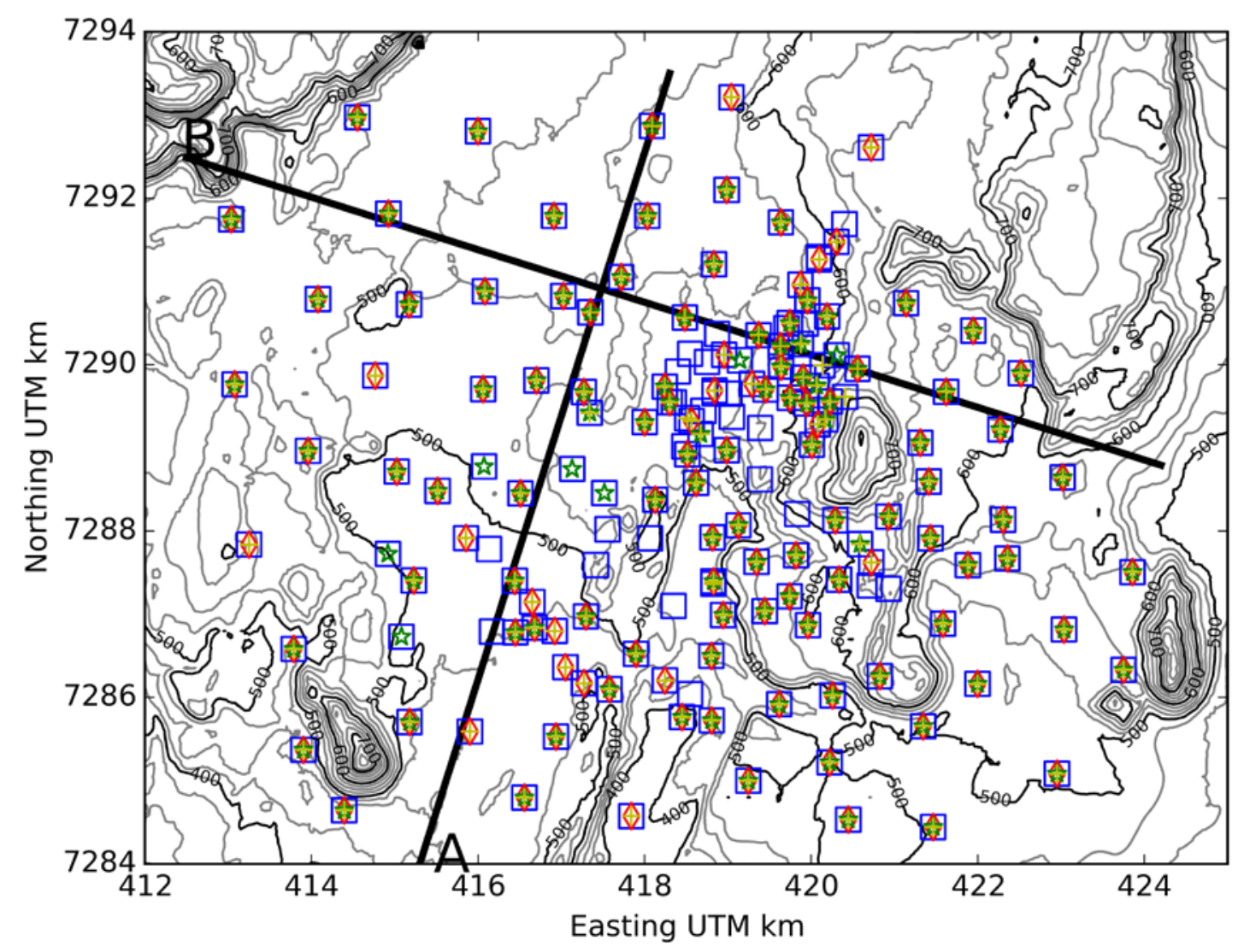

149

150 Figure 1. Topographic map and MT station locations at Krafla. All MT station locations are 151 shown as blue squares. Stations used with WSINV3DMT are shown by red diamonds; stations 152 used with EMGeo are show as green stars; stations used with MT3Dinv are shown as yellow 153 crosses. Black lines (A, B) show sections used for discussion of recovered models in Section 5.

155 Figure 1 shows the topographic relief and locations of MT soundings. In total, 167 MT soundings

156 were collected at Krafla. These MT data were collected in three major field campaigns: by a

157 group from Duke University during the summers of 2004 and 2005 (108 soundings); by Moscow

158 State University, and Iceland Geosurvey in the summers of 2006 (55 soundings); and 2008 (5

159 soundings). All soundings were collected with equipment from Phoenix Geophysics Ltd., and

160 processed using the software package provided with the instruments. The data-processing

161 package includes the Gamble et al. (1979) remote reference routine. The subsets of the Krafla 
163 Figure 1 as red diamonds, green stars, and yellow crosses, respectively.

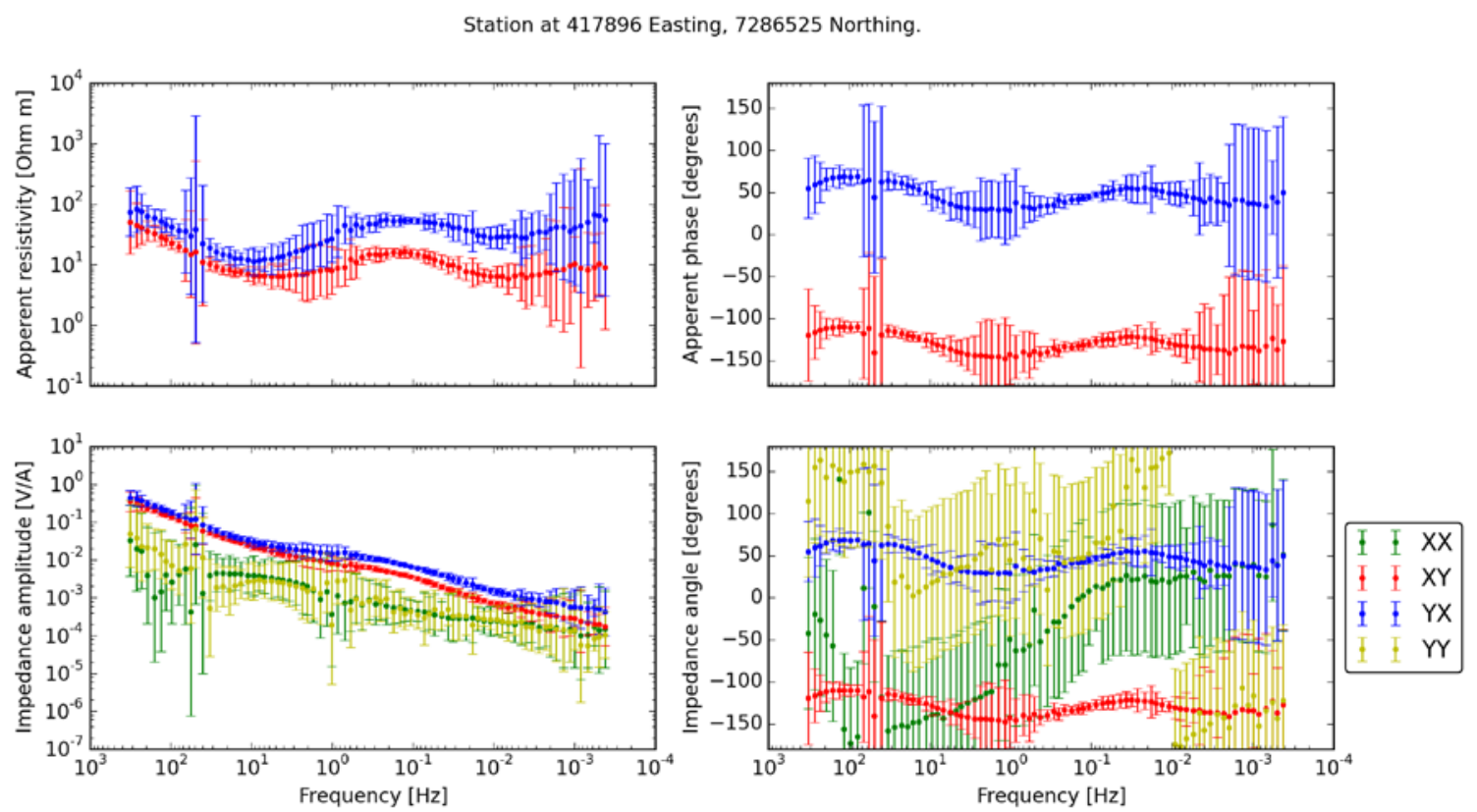

165 Figure 2. An example of MT data at Krafla. At the highest frequencies, parallel apparent resistivity and phase curves of Zxy and Zyx components are conspicuous indicating that Earth resistivity is more or less one dimensional in the upper part of the geological section. This finding is also confirmed with the on-diagonal impedance elements, which are significantly smaller in amplitude (at least an order of magnitude) until reaching a frequency of $0.1 \mathrm{~Hz}$. The frequencies are plotted decreasing from left to right.

Figure 2 shows an example of an MT sounding at Krafla. The responses at frequencies above

173 approximately $10 \mathrm{~Hz}$ exhibit a 1D character, where the off-diagonal soundings Zxy and Zyx are

174 parallel and equal in amplitude. The diagonal elements Zxx and Zyy (the green and yellow

175 impedance curves, respectively) are considerably lower in amplitude than the off-diagonal

176 elements for most frequencies. Our assessment of the quality of the Krafla dataset is that it

177 contains a considerable amount of noisy data, increasing the complexity of the inversion process. 
180 The Hengill geothermal area is located in SW Iceland, at the triple junction of the Reykjanes

181 Peninsula, the Western Volcanic Zone, and the South Iceland Seismic Zone. The active fissure

182 swarm in the area has a SW-NE orientation, coincident with the main volcanic fissure that cuts

183 through the Hengill volcano. Again, for a more detailed discussion of the geology, see

184 Gasperikova et al. (2015) (this issue).

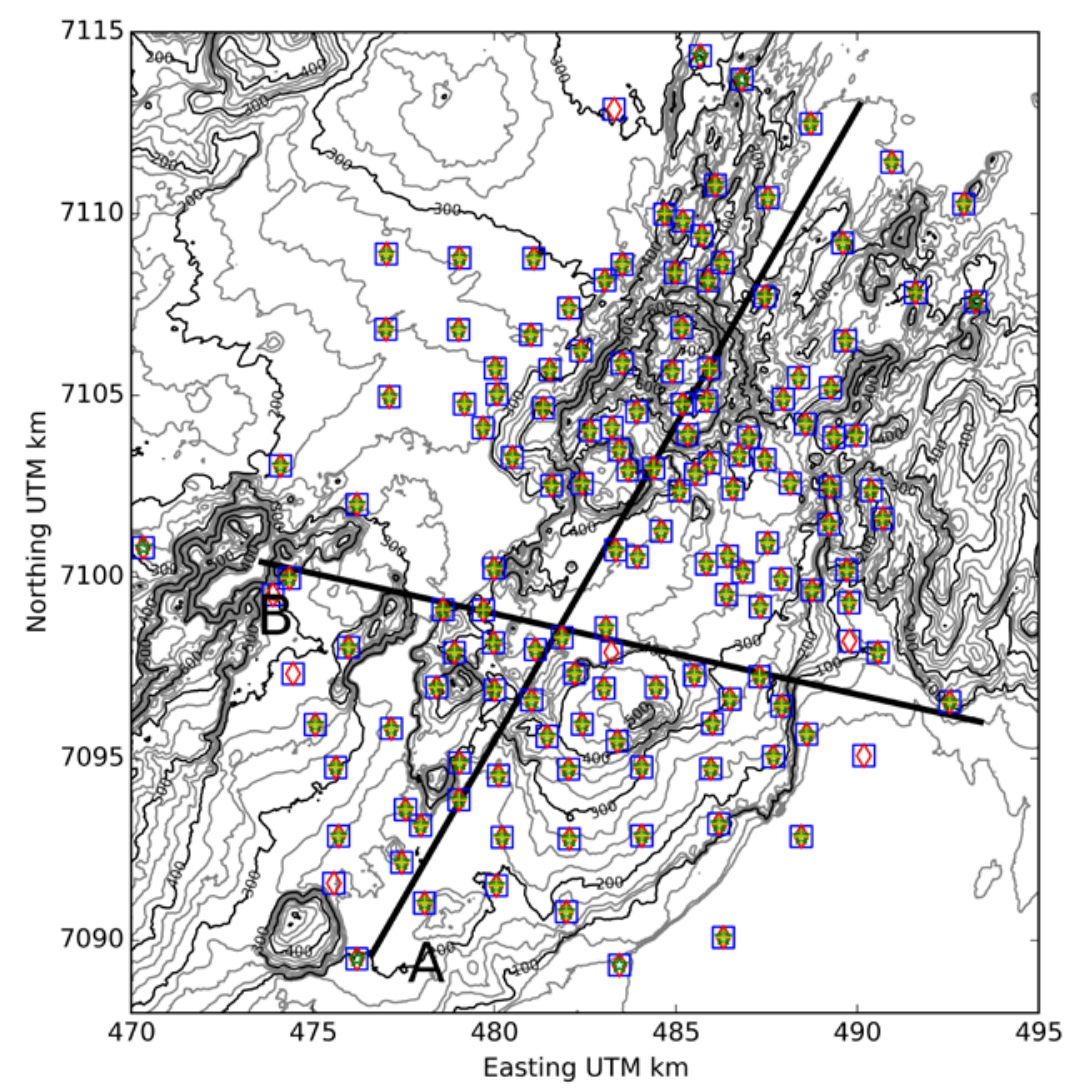

185

186 Figure 3. Topographic map and MT station locations at Hengill. All MT station locations are shown as blue squares. The locations used with WSINV3DMT are shown by red diamonds, locations used with EMGeo are show as green stars, and locations used with MT3Dinv are shown as yellow crosses. Black lines (A, B) show sections used for discussion of recovered models in 190 Section 5. 
193 Figure 3 shows the topographic relief and locations of MT soundings at Hengill. The original MT

194 data were acquired by the Iceland Geosurvey in four collection campaigns (Árnason et al., 2010);

195 for this study, a total of 144 soundings were available for the inversion, shown as blue squares in

196 Figure 3. All the soundings were collected with equipment from Phoenix Geophysics Ltd. In

197 some cases, only the electric fields were collected, and the magnetic field data at a nearby site

198 were used for estimating the impedance tensor. The data were processed with software provided

199 with the instruments, which included a remote reference processing routine (Gamble et al., 1979).

200 Overall, data quality is very good, better than the Krafla dataset. One example of the impedance

201 and apparent resistivity curves are shown in Figure 4. This example highlights how, like the

202 Krafla example, the off-diagonal elements Zxy and Zyx are equal in amplitude for frequencies

203 above $10 \mathrm{~Hz}$. The diagonal elements Zxx and Zyy are found to be considerably lower in

204 amplitude than the off-diagonal elements for most frequencies.

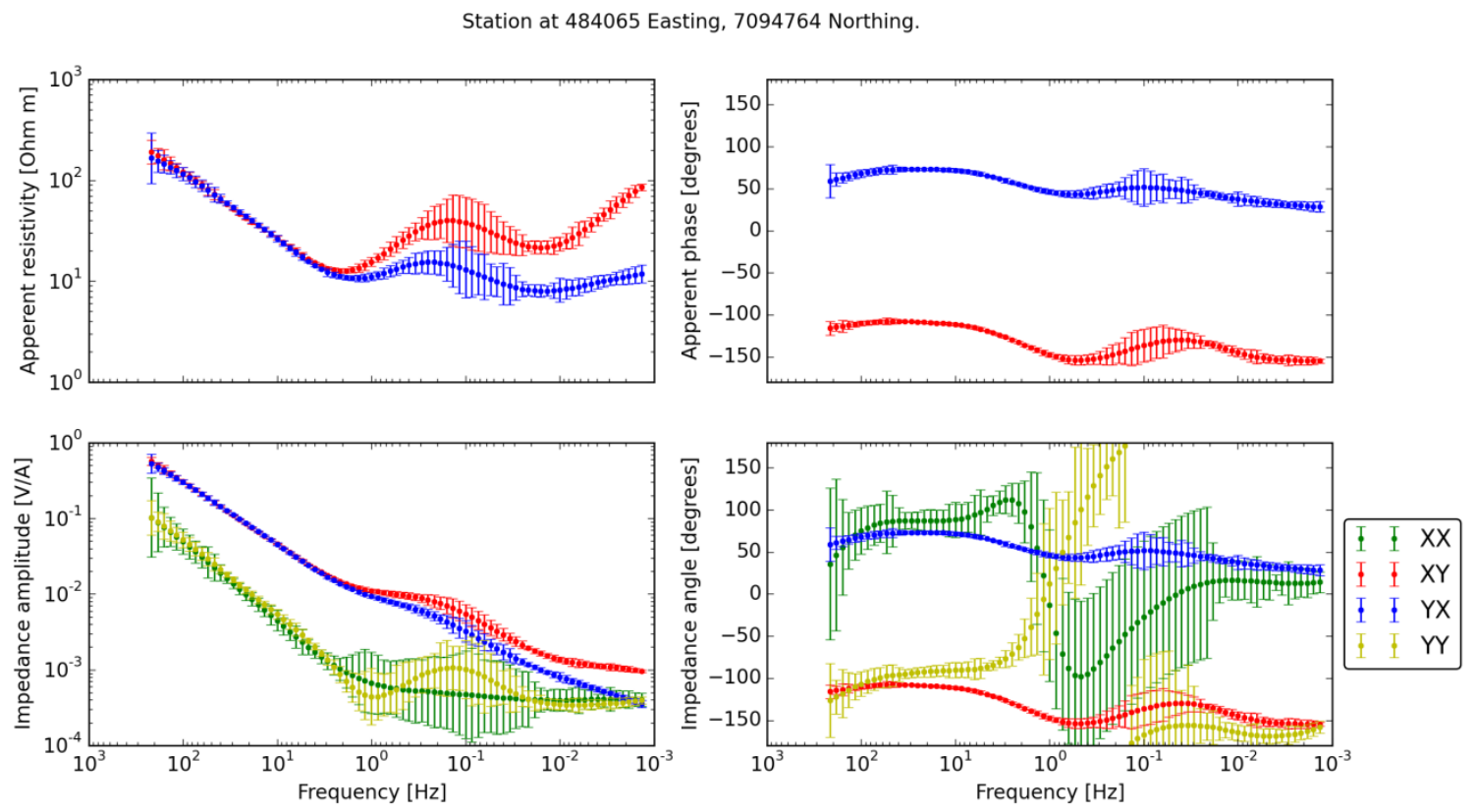

206 Figure 4. An example of MT data from Hengill The data show a 1D character at the highest 
208 impedance elements are significantly smaller in amplitude (an order of magnitude) for most of 209 the frequency range.

\section{Inversion algorithms}

213 The goal of MT inversion is to construct a resistivity model that produces responses that fit the

214 observed data within a given tolerance. Owing to the non-uniqueness of the 3D MT inversion

215 problem, we must regularize the procedure by enforcing constraints on an acceptable model. This

216 is commonly done by requiring the model to change gradually between adjacent cells: by using a

217 reference model to enforce a priori information; and/or by restricting the range of resistivity

218 values that can be used in the inverse solution. The choice and implementation of the constraints

219 will influence the recovered model, which also needs to be designed considering prior knowledge

220 of the problem and the geological settings at any given place. The inversion problem can be

221 described with the generalized objective function

$222 \phi=\phi_{d}+\beta \phi_{m}$,

224 where $\boldsymbol{\phi}$ is the overall objective function, $\boldsymbol{\phi}_{\boldsymbol{d}}$ is a data misfit measuring the difference between

225 the predicted and observed data, $\boldsymbol{\phi}_{\boldsymbol{m}}$ is the model misfit introducing constraints to the model, and

$226 \boldsymbol{\beta}$ is the trade-off parameter controlling relative significance between the data and model misfits

227 in the overall objective function. Estimating an appropriate value for $\boldsymbol{\beta}$ is important, because a

228 large $\boldsymbol{\beta}$ will poorly fit the data, due to the heavy influence of the model constraint introduced in

229 the model misfit term in Equation ( 4 ), whereas a small $\boldsymbol{\beta}$ will fit the data better by requiring

230 more structure in the recovered model because of the lower importance of the model constraints 
231 (Oldenburg and $\mathrm{Li}, 2005$ ). A common way of estimating trade-off parameters is to use a cooling

232 scheme: the inversion is started with a large $\boldsymbol{\beta}$, which then gradually decreases in value as the

233 inversion progresses. The inversion progresses to a new $\boldsymbol{\beta}$ only after effectively converging for

234 the previous one, when the inversion process for a fixed $\boldsymbol{\beta}$ is found to be making minimal

235 progress in further reducing the misfit of the objective function. The global goal of the inversion

236 is to fit the data to a pre-specified tolerance, assuming that the estimations of data errors in the

237 data misfit term are valid.

238 During each iteration step of the inversion, multiple forward calculations of the resistivity model

239 are required. The number of times the forward problem needs to be solved depends on the

240 number of unique frequencies and number of model evaluations required at each inversion

241 iteration step. The complexity of each of these forward solutions will depend on the number of

242 cells in the mesh and the efficiency of the solver algorithm.

243 Note that 3D MT inversion algorithms can be implemented in different ways; Siripunvaraporn

244 (2011) gives a good overview of common numerical algorithms used in MT. Parallelization of

245 the inversion algorithms is also an important factor in their speed and usability. Distributing the

246 calculations over multiple CPU threads significantly reduces the time required to solve the

247 problem and allows for bigger systems to be solved by increased CPU power and shared memory

248 of the threads, respectively. Running on multiple CPUs significantly improves efficiency, making

249 the solution of larger and more realistic inversion problems feasible.

250 In this study, we use three different inversion codes to invert data from the Hengill and Krafla

251 geothermal areas in Iceland. These inversion codes are discussed briefly below; more detailed

252 descriptions of these codes can be found in given references. 
255 The WSINV3DMT inversion code uses a data-space variant of the minimum-structure Occam

256 inversion approach. Following Siripunvaraporn et al. (2005), the objective function to be

257 minimized is

258

$U(m, \lambda)=\left(m-m_{0}\right)^{T} C_{m}^{-1}\left(m-m_{0}\right)+\lambda\left\{(d-F[m])^{T} C_{d}^{-1}(d-F[m])-X^{* 2}\right\}$,

259

260 where $\boldsymbol{m}$ and $\boldsymbol{m}_{0}$ are the resistivity model and the prior resistivity model, respectively, $\boldsymbol{C}_{m}$ is the

261 model covariance matrix, which contains information about model regularization, $\boldsymbol{d}$ is observed

262 data, $\boldsymbol{F}[\mathrm{m}]$ is forward calculated predicted data, $\boldsymbol{C}_{d}$ is the data covariance matrix containing

263 information about the data uncertainties, $X^{*}$ is the desired level of misfit, and $\lambda$ is the trade-off

264 parameter. A parallelized solution strategy shown in Equation ( 5 ) is described in

265 Siripunvaraporn and Egbert (2009). WSINV3DMT assumes a flat surface in the model

266 discretization; thus, one cannot include topography.

\subsection{EMGeo}

269 The EMGeo inversion code uses a nonlinear conjugate-gradient optimization scheme (Newman

270 and Alumbaugh, 2000) with an approximate Hessian as a pre-conditioner (Newman and Boggs,

271 2004). The objective function is a weighted sum of the data misfit, and includes a model

272 regularization that allows for smooth model reconstructions along with a reference model. Here,

273 the objective function is given as

$274 \varphi=\sum_{n=1}^{2 N}\left[\frac{\left(z_{n}^{o b s}-z_{n}\right)}{\epsilon_{n}}\right]^{2}+\lambda\left(\boldsymbol{m}-\boldsymbol{m}_{r e f}\right)^{T} \boldsymbol{W}^{T} \boldsymbol{W}\left(\boldsymbol{m}-\boldsymbol{m}_{\boldsymbol{r e f}}\right)$, 
275 where $Z_{n}^{\text {obs }}$ and $Z_{n}$ are the observed data and predicted data for the $\mathrm{n}^{\text {th }}$ data, respectively, $\epsilon_{n}$ is the

276 data misfit of the $\mathrm{n}^{\text {th }}$ data, and $\boldsymbol{W}$ consists of a finite difference approximation of the model

277 Laplacian operator. In Equation (6), the regularization parameter $\lambda$ controls model regularization

278 in the objective function. This algorithm has proven successful in previous 3D MT imaging

279 studies of geothermal fields (see Newman et al., 2008).

280 During the inversion, the regularization parameter $\lambda$ is decreased; initially a large fixed value is 281 used, which decreases incrementally during the inversion. In other words, as the inversion 282 progresses, the weight of the model regularization decreases in Equation ( 6 ), allowing for 283 rougher structures to be incorporated into the model, as needed. The inversion runs until an 284 appropriate data misfit is achieved. The inversion code is optimized to run on a large number of 285 cores, in which calculations can be distributed and solved simultaneously to reduce the time to 286 solution.

\subsection{MT3Dinv}

The MT3Dinv inversion code uses an iterative Gauss-Newton procedure to solve the inverse

290 problem (Farquharson et al., 2002). It searches for a minimum of the objective function

$291 \phi=\left\|W_{d}\left(F(m)-d^{o b s}\right)\right\|_{2}^{2}+\beta \sum_{k}\left(\alpha_{k}\left\|W_{k}\left(m-m_{r e f}\right)\right\|_{2}^{2}\right)_{k=s, x, y, z}$

293 where $\boldsymbol{m}$ is a vector expressing the model parameters, $\boldsymbol{m}_{r e f}$ is the reference model, $\boldsymbol{W}_{d}$ is the

294 diagonal matrix containing the data uncertainties, $\boldsymbol{d}^{\text {obs }}$ is the observed data, and $\boldsymbol{F}(\boldsymbol{m})$ is the

295 forward modeling operator that produces a response for a given resistivity model. $\boldsymbol{\beta}$ is the trade- 
296 off parameter between the data and model misfits, $\boldsymbol{W}_{S}$ is a diagonal damping matrix, and

$297 \boldsymbol{W}_{x}, \boldsymbol{W}_{y}, \boldsymbol{W}_{z}$ are the first order finite-difference matrices in the x, y, and z directions in the

298 model, respectively. $\alpha_{s}, \alpha_{x}, \alpha_{y}$ and $\alpha_{z}$ are scaling factors that control the importance of prior

299 model information and constraints in the objective function, described by the model misfit in

300 Equation ( 7 ). Further constraints can be introduced into model, where bounds on the allowed

301 conductivity values can be implemented. This can be done globally for all the cells in the model,

302 where all values are within a conductivity range; or locally for each cell, where each cell has its

303 own conductivity bound.

304 The regularization parameter $\boldsymbol{\beta}$ is decreased during the inversion process. Starting with a large

305 value for $\boldsymbol{\beta}$, the optimization problem is solved iteratively at each step, until either the

306 appropriate misfit error has been achieved or the maximum number of iterations is reached. Once

307 finished, the model is updated, $\boldsymbol{\beta}$ is decreased, and the process is repeated until a satisfactory data

308 misfit is reached. An option is implemented in the code to allow for $\boldsymbol{m}_{\text {ref }}$ to be updated for each

309 new $\boldsymbol{\beta}$ using the resulting model from the previous iteration. This can increase the convergence

310 rate of the inversion problem. The code is parallelized to distribute the solution of the MT source

311 fields for each frequency on different compute cores, significantly increasing the speed of the

312 inversion process.

313

314 4. Description of inversions

315 Owing to the non-uniqueness of the inversion procedure, the same data set can be fit to the same

316 degree with different resistivity models. Thus model interpretation is best done in the context of, 
317 or in conjunction with, other available information about a region, e.g., geological information,

318 other geophysical data, and borehole data. Running multiple inversions with different settings

319 and inputs, and using multiple starting models is considered the current best practice. It provides

320 a fair control on which structures and features are required by the data and which are not (and

321 should be considered as artifacts).

322 It is usually impractical to invert data for all measured frequencies, since computational

323 requirements for the inversion codes are highly dependent on the number of frequencies. Care

324 must be taken to select frequencies that correspond with the desired depth of investigation, and to

325 remove noisy data by either excluding them from the data set or by assigning larger errors. There

326 are, in fact, many important and delicate steps regarding data input that need to be taken in order

327 for an inversion to run effectively. These include understanding the data that are used in the

328 inversion, providing consistent data with regard to the units that the inversion algorithm requires,

329 ensuring that the data vectors align with the coordinate axes used in the algorithm, and ensuring

330 that the assumption of time dependency $e^{-i \omega t}$ or $e^{i \omega t}$ for the data matches that of the algorithm.

331 If these steps are not addressed, the inversion will not be able to function properly and will not

332 converge to a satisfactory solution. Siripunvaraporn (2011) discusses these issues and provides

333 guidelines for addressing them within the inversion process.

334 Mesh design also plays an important role in the solution of the problem. When discretizing the

335 Earth, the tradeoff between accuracy, which generally requires a large number of cells, and

336 efficiency, which generally requires reducing the computational requirements, needs to be

337 considered. Furthermore, it is important to discretize the Earth such that numerical errors are

338 minimized by using small enough cells, but without exceeding the limits of the memory and CPU

339 power available for solution of the inverse problem. 
340 Another source of numerical errors may arise from the mesh boundaries. It is common to assume

341 1D or 2D field behavior as boundary conditions. To account for such conditions, the core mesh

342 (the part of a model that contains the data and has uniform horizontal cell sizes) is padded with

343 cells that progressively increase in size away from the core mesh. This minimizes the influence of

344 the boundary conditions in calculations of predicted MT soundings, data sensitivities, and

345 functional gradients.

346 In practice, running an inversion also requires the user to set parameters that control the weights

347 of the different terms in the objective function, as well as other settings required by numerical

348 solvers and optimization algorithms employed within the inversion code. Having a basic

349 understanding of what these settings are and how they may affect the results is important, to

350 improve the interpretation of the model. It is important to be mindful of the inversion process

351 setup, data quality; discretization and inversion settings. For example, when a coarse mesh and

352 low frequencies are used, detail structures in the near surface cannot be resolved. Similarly,

353 smooth regularization doesn’t allow for sharp interfaces between resistivity units. In the

354 following section, we will discuss the setups of the inversion codes used in this study. The same

355 initial static shift corrected data set was available to the users, but each user selected a subset of

356 the data (both locations and frequencies) and assigned errors as appropriate for the particular

357 code. Figure 1 and Figure 3 show the MT stations used for Krafla and Hengill, respectively. No

358 restrictions were enforced in terms of the starting model choices, the details of how the inversions

359 were run, or the constraints imposed on the different inversion codes.

360 Table 1. Inversion parameters and settings of the inversion codes used for the Hengill and Krafla 361 MT datasets.

\begin{tabular}{|l|l|l|l|}
\hline & WSINV3DMT & EMGeo & MT3Dinv \\
\hline Hengill inversions
\end{tabular}




\begin{tabular}{|c|c|c|c|c|}
\hline \multirow[t]{2}{*}{ Data } & Frequencies & $\begin{array}{l}10^{2}-10^{-3}, 5 \text { per decade } \\
26 \text { in total }\end{array}$ & $\begin{array}{l}10^{2.3}-10^{-2}, 3 \text { per decade } \\
15 \text { in total }\end{array}$ & $\begin{array}{l}10^{2}-10^{-2.3}, 3 \text { per decade } \\
16 \text { in total }\end{array}$ \\
\hline & Number stations & 144 & 138 & 132 \\
\hline \multirow[t]{2}{*}{ Core mesh } & $\begin{array}{l}\text { Number of cells } \\
x-y-z\end{array}$ & $39-47-18$ & $79-93-50$ & $79-94-67$ \\
\hline & Smallest cell $[\mathrm{m}]$ & $500 \times 500 \times 8$ & $250 \times 250 \times 50$ & $250 \times 250 \times 50$ \\
\hline \multicolumn{2}{|c|}{ Initial starting model [Ohm-m] } & 50 & 30 & 16 \\
\hline \multicolumn{5}{|c|}{ Krafla inversions } \\
\hline \multirow[t]{2}{*}{ Data } & Frequencies & $\begin{array}{l}10^{2}-10^{-3}, 5 \text { per decade } \\
26 \text { in total }\end{array}$ & $\begin{array}{l}10^{2.3}-10^{-2}, 3 \text { per decade } \\
15 \text { in total }\end{array}$ & $\begin{array}{l}10^{2}-10^{-2.6}, 5 \text { per decade } \\
24 \text { in total }\end{array}$ \\
\hline & Number stations & 122 & 130 & 116 \\
\hline \multirow[t]{2}{*}{ Core mesh } & $\begin{array}{l}\text { Number of cells } \\
x-y-z\end{array}$ & $37-33-18$ & $62-51-84$ & $49-45-59$ \\
\hline & Smallest cell [m] & $300 \times 300 x 8$ & $250 \times 250 \times 50$ & $250 \times 250 \times 50$ \\
\hline \multicolumn{2}{|c|}{ Initial starting model [Ohm-m] } & 20 & 30 & 20 \\
\hline
\end{tabular}

\subsection{WSINV3DMT}

364 Some of the key parameters used in the WSINV3DMT inversion for the Hengill and Krafla areas 365 are described in

366 Table 1. The mesh used in the Hengill area is based on a local reference frame that is rotated $30^{\circ}$

367 clockwise from true North. The mesh used for Krafla is on a frame parallel to the UTM grid.

368 Padding cells were added to the sides of the core mesh, where the side padding was incrementally

369 increased in size by a factor ranging from 1.2 to 1.8. The mesh contains 28 cells in the vertical

370 direction, growing by a factor of 1.4 from thickness of $8 \mathrm{~m}$ to $65 \mathrm{~km}$ at the bottom mesh 
371 boundary. In both areas, no topography is incorporated within the model. Data errors used in the

372 covariance matrix were set to the variances provided in the EDI files, but a minimum uncertainty

373 error floor was set at 3\% and 5\% of a given data value for Hengill and Krafla data, respectively.

374 Each inversion was implemented in stages, with the observed data (frequencies and stations) and

375 mesh kept constant at all stages but the reference model was updated. Each stage consisted of five

376 inversion iterations, wherein both the reference and starting models were set as the resulting

377 model from the previous stage. For both the Hengill and Krafla inversions, five stages were

378 required for the procedure to converge and to recover a satisfactory model.

\subsection{EMGeo}

381 Some of the key parameters used in the implementation of the EMGeo inversion code for the

382 Hengill and Krafla areas are given in Table 1.In both areas, the mesh is parallel to the UTM grid.

383 The size of the padding cells that surrounded the core mesh progressively increases by a factor of

3841.2 away from the core mesh, until the lateral model boundary is approximately five skin depths

385 away from the core mesh. A vertical cell thickness of $50 \mathrm{~m}$ is used in the topographic range; this

386 thickness was continuously increased to $500 \mathrm{~m}$ and then kept constant to $10 \mathrm{~km}$ depth. Below that

387 depth, the cell thickness grows by a factor of 1.2. The model extends $100 \mathrm{~km}$ above and below

388 sea level, with approximately 20 cells above topography, which were included in the model

389 domain. For both areas, the inversion is constructed with the same inversion input parameters.

390 EMGeo employs a Laplacian smoothness model regularization and a cooling scheme for the

391 trade-off parameter, as described previously. A global bound is used, forcing the resistivity

392 values to be between 2000 to 1 Ohm-m. The data misfit used is $5 \%$ of the given data value. In

393 order to properly fit the full bandwidth of data, the inversion procedure is sequenced into two 
394 stages as described in detail by Lindsey and Newman (2014). In the first stage, only frequencies

395 below $1 \mathrm{~Hz}$ were inverted on a coarse mesh (smallest core cell size of $500 \mathrm{~m} \times 500 \mathrm{~m} \times 50 \mathrm{~m}$ ).

396 After fitting the low frequency data, the resulting coarse model was re-parameterized onto a fine

397 mesh (smallest core cells size of $250 \mathrm{~m} \times 250 \mathrm{~m} \times 50 \mathrm{~m}$ ), which was sequentially used as a

398 starting model for the second stage. In the second stage, the data from the full frequency range

399 were used.

400

$401 \quad 4.3$ MT3Dinv

402 Some of the key parameters used in the implementation of MT3Dinv inversion code for the

403 Hengill and Krafla areas are given in

404 Table 1. In both areas, the mesh is parallel to the UTM grid. Horizontally, the padding cells

405 around the core mesh are progressively increased by a factor of 1.4 away for the core mesh.

406 Within the core mesh, the cell thickness was incrementally increased with depth from $50 \mathrm{~m}$ to

$407650 \mathrm{~m}$ at $10 \mathrm{~km}$ depth, beyond which the thickness grew by a factor of 1.4. In the topographic

408 region, a vertical thickness of $50 \mathrm{~m}$ was used to discretize the topographic relief in the areas. The

409 inversions were set up in similar ways for both areas. Global bounds were used to force all the

410 values of the cells in the model to be between 5000 and $0.1 \mathrm{Ohm}-\mathrm{m}$, the model regularization

411 parameters $\alpha_{x, y, z}$ were set to 1 , and $\alpha_{s}$ was set to $10^{-7}$, much smaller relative to $\alpha_{x, y, z}$, in order to

412 force smoothness in the recovered model. The data uncertainties used for the inversion were set

413 as $10 \%$ of the data value, plus an error floor set to be $5 \%$ of the mean for the off-diagonal

414 elements at each frequency. 
415 To increase the efficiency of the inversion procedure, it was conducted in stages, with the mesh

416 resolution coupled with the subsample of the frequencies used in the data analysis. For the

417 Hengill inversion, the procedure was conducted in three stages, as described in Rosenkjaer and

418 Oldenburg (2012). The workflow for the Krafla data set contained only two stages. In the first

419 stage, a coarse mesh (core cell size of $500 \mathrm{~m} \times 500 \mathrm{~m} \times 75 \mathrm{~m}$ ) was used to invert 12 frequencies

420 below $10 \mathrm{~Hz}$. The resulting model was re-parameterized onto a finer mesh (core cell size of cell

421 size $250 \mathrm{~m} \times 250 \mathrm{~m} \times 50 \mathrm{~m}$ ) and the full frequency range in Table 1 was used. During inversion

422 using the coarse mesh, the reference model was not updated at the start of each new $\boldsymbol{\beta}$ step;

423 however, during the inversion with the fine mesh, the reference model was updated at that point.

\section{Comparison of Resistivity Models}

426 Here we describe a novel set of metrics to evaluate structural features in 3D MT resistivity

427 models and then use these metrics to interrogate the retrieved models for Krafla and Hengill. We

428 propose using gradient and Laplacian operators, along with resistivity models, to add a

429 quantitative analysis to the comparison of the recovered models. We further propose to use a

430 cross-gradient comparison between two models to map out the structural correlation between the

431 respective models. The goal of comparing inversion results in this way is to identify robust

432 resistivity structures that are required by the data and that are therefore better suited for

433 geological and petrological interpretation. All model analyses and visualization were done with

434 Python scripts, using a combination of Numpy (Oliphant, 2007) and Visualization Toolkit (VTK)

435 (Schroeder et al., 2006). Our ultimate goal is to assess and identify common features in the 
436 models. These common features are discussed and interpreted in terms of geology in

437 (Gasperikova et al., 2015) (this issue).

\subsection{Description of comparison procedure}

439 All the inversions were run on different meshes, so in order to conduct comparisons, we had to

440 transfer all the models to a common grid. For the comparison grid, we used only the areas in the

441 models covered by data with topography incorporated. Calculations of gradient and Laplacian

442 parameters were performed on the original meshes, and then transformed to the comparison grid

443 with an inverse-distance weighted interpolation scheme. Because inversions using WSINV3DMT

444 did not include topography, we needed an additional processing step during the regridding

445 process for the WSINV3DMT models, to shift cells in the vertical direction.

\section{$446 \quad$ 5.1.1 Definitions of the parameters}

447 We propose to extract structural information from 3D MT models that is especially useful when

448 comparing two models. The approach uses a series of metrics based on the gradient, cross-

449 gradient and Laplacian operators. In the derived calculations below, we

450 use $m=\log _{10}$ (model resistivity).

451 The model gradient is calculated as

$452 \nabla \boldsymbol{m}=\left[\frac{\partial m}{\partial x} \hat{\boldsymbol{\imath}}, \frac{\partial m}{\partial y} \hat{\jmath}, \frac{\partial m}{\partial z} \widehat{\boldsymbol{k}}\right]$,

453

454 where the gradient vector maps the change in resistivity of adjacent cells scaled by the distance

455 between their cell centers in the three orthogonal directions. The gradient calculations of the

456 models use a cell-centered discretization implemented in VTK. For visualization of the gradient,

457 we use the magnitude of the gradient, 


$$
|\nabla m|=\sqrt{\left(\frac{\partial m}{\partial x}\right)^{2}+\left(\frac{\partial m}{\partial y}\right)^{2}+\left(\frac{\partial m}{\partial z}\right)^{2}}
$$

459 where the gradient magnitude is a scalar of the square root of the sum of the squared three

460 orthogonal gradient components. It maps the magnitude of the change in resistivity, irrespective

461 of the resistivity increasing or decreasing.

462 The Laplacian is calculated as

$463 \quad \nabla^{2} m=\left(\frac{\partial^{2} m}{\partial x^{2}}+\frac{\partial^{2} m}{\partial y^{2}}+\frac{\partial^{2} m}{\partial z^{2}}\right)$.

465 The Laplacian gives an estimate of the curvature of the resistivity in all directions, with a positive 466 value correlating with a positive (upward) curvature, and similarly a negative value correlating 467 with a negative (downward) curvature in the model. Related to the resistivity structure, a positive 468 Laplacian correlates to features in which the resistivity increases at a faster rate compared to its 469 surroundings, and a negative Laplacian corresponds to features in which the resistivity decreases 470 at a faster rate compared to its surroundings.

471 To compare two resistivity models $\left(m_{1}\right.$ and $\left.m_{2}\right)$ directly, we use the cross-gradient (cross

472 products of gradients) between the two models

$473 \nabla m_{1} \times \nabla m_{2}=\left[\left(\frac{\partial m_{1}}{\partial y} \frac{\partial m_{2}}{\partial z}-\frac{\partial m_{1}}{\partial z} \frac{\partial m_{2}}{\partial y}\right) \hat{i}+\left(\frac{\partial m_{1}}{\partial x} \frac{\partial m_{2}}{\partial z}-\frac{\partial m_{1}}{\partial z} \frac{\partial m_{2}}{\partial x}\right) \hat{j}+\left(\frac{\partial m_{1}}{\partial x} \frac{\partial m_{2}}{\partial y}-\frac{\partial m_{1}}{\partial y} \frac{\partial m_{2}}{\partial x}\right) \hat{k}\right]$,

475 where the cross-gradient is a vector mapping the differences of the gradients of the two models.

476 Since the result of Equation ( 11 ) is a vector, for ease of viewing and illustration, we use its

477 normalized magnitude,

$478 \quad \operatorname{Norm}\left(\nabla m_{1} \times \nabla m_{2}\right)=\frac{\left|\nabla m_{1} \times \nabla m_{2}\right|}{\left|\nabla m_{1}\right|\left|\nabla m_{2}\right|}$. 
479 We implemented a minimum threshold on $\left|\nabla m_{1}\right|$ and $\left|\nabla m_{2}\right|$ to avoid the problem of dividing by 480 a number close to 0 . This problem occurs where there is little or no structure present in one or 481 both models. The threshold was set to be 1.5 standard deviations from the mean of the 482 magnitude. Values that fall below this threshold are not considered (i.e., value set to NaN "not a 483 number”), which forces the result for these cells from Equation ( 12 ) to be NaN, as well as 484 illustrating that the normalization is ill-posed. The aim of using the parameters discussed above is 485 to highlight structures recovered in the resistivity models and help with a structural comparison 486 between the models. Illustration of the quantitative power of these metrics is discussed using 487 idealized synthetic examples in Appendix A.

\section{$488 \quad 5.2$ Krafla}

489 Cross-sections along the profile shown as black lines in Figure 1 resulting from the inversion 490 codes described in Sections 3.1, 3.2, and 3.3 are shown in Figure 5(a), (d), and (g), respectively. 491 Corresponding gradient magnitudes of each resistivity model are shown in Figure 5(b), (e), and 492 (h), and the Laplacians of these models are shown in Figure 5(c), (f), and (i). 

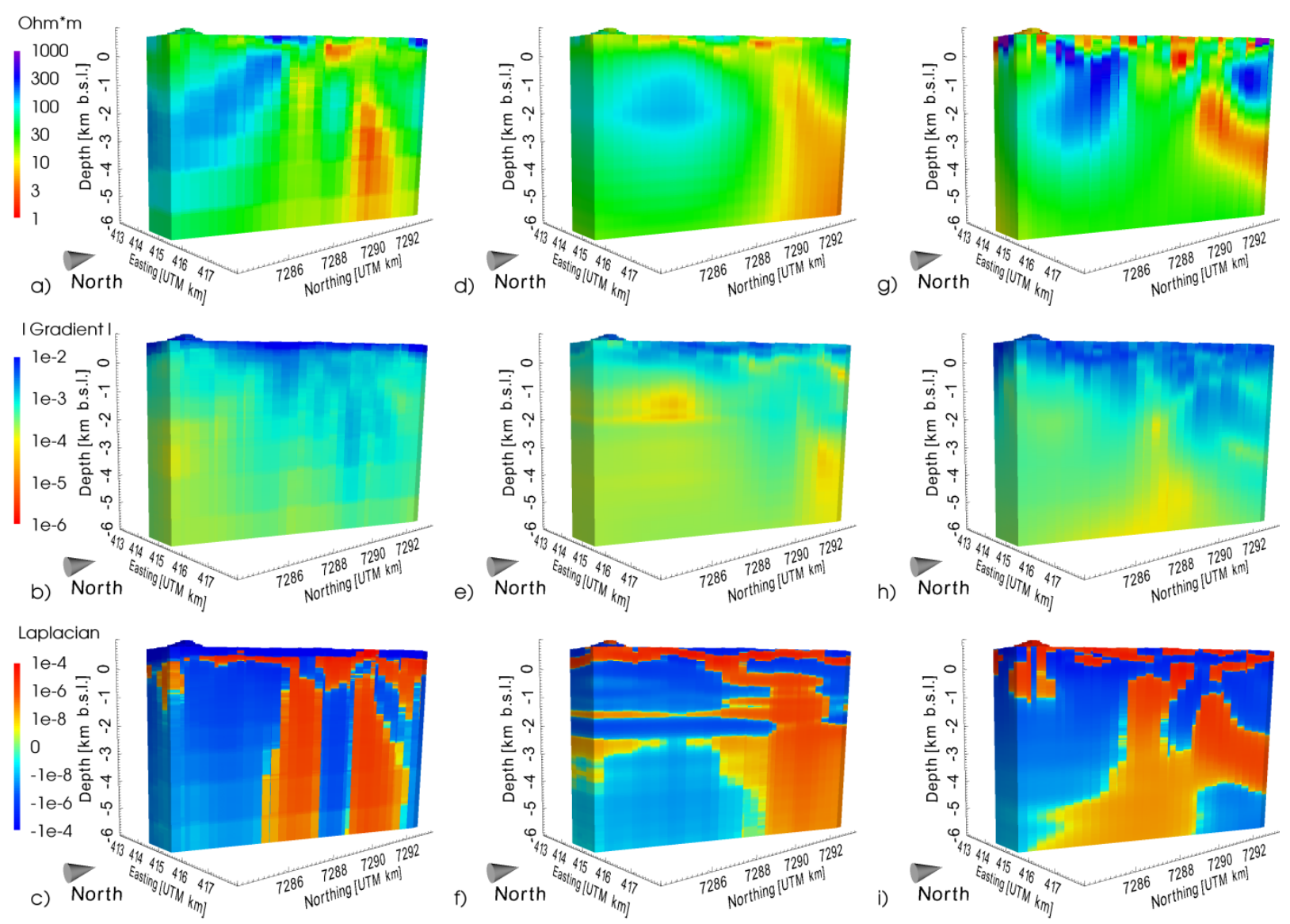

494 Figure 5. A cross section along the main fissure in Krafla: (a), resistivity, (b) gradient, and (c) 495 Laplacian are for results obtained with WSINV3DMT; (d) resistivity, (e) gradient, and (f) 496 Laplacian are for results obtained with EMGeo; and (g) resistivity, (h) gradient, and (i) Laplacian 497 are for results obtained with MT3Dinv.

499 In the near surface, the WSINV3DMT model in Figure 5(a) is resistive ( $>200$ Ohm-m), but there

500 is a discontinuous conductive region beneath (ranging from 10 to 25 Ohm-m), which connects

501 upward to the surface in places. A resistive body (100-500 Ohm-m) is seen in the southern part of

502 the model (centered at $7286 \mathrm{~N}$, depth of $2 \mathrm{~km}$ ), but two conductive vertical structures (both less

503 than 10 Ohm-m) are observed in the central (7289 N) and northern (7291 N) part of the section.

504 The northern conductive structure is more conductive, larger and discernible. In Figure 5b, the

505 magnitude of the gradient is highest in the near surface, and the vertical contacts are seen as high 
506 amplitude vertical stripes. The Laplacian in panel (c) reveals two distinct conductive bodies as

507 upward curved pillars with a clear permutation of the curvature at the locations of the conductive 508 structures in panel (a).

509 The surface resistivity of the EMGeo model in Figure 5(d) is conductive above the resistive core 510 in the southern part of the section $(7285 \mathrm{~N})$, connecting to a fairly continuous layer with

511 resistivity less than $10 \mathrm{Ohm}-\mathrm{m}$, though there are narrow gaps of relatively higher resistivity. A

512 conductive zone $(<15 \mathrm{Ohm}-\mathrm{m})$ is present in the northern part (centered around $7291 \mathrm{~N})$ of the

513 section. This conductive zone comes within $1 \mathrm{~km}$ of the surface and has a large base at depth.

514 The model gradient and Laplacian in panels (e) and (f) illustrate the dominant resistive structure

515 to the south and the conductive zone to the north. In panel (f), horizontal features are revealed

516 that are not apparent in the resistivity model in panel (d). We have determined that these are

517 artifacts resulting from the reparameterization of the model (coarse grid to fine grid), as discussed

518 in Section 4.2.

519 The MT3Dinv resistivity model in Figure 5(g) shows a resemblance to the model in panel (a);

520 however, the model in panel (g) has fewer vertical structures, and the conductive overburden is

521 more scattered. There is a dominant resistivity structure in the south and a conductive zone in the

522 north (centered in similar locations as in panels (a) and (d)), extending from a depth of $5 \mathrm{~km}$ to

523 the near surface. In the center of the section (at $7290 \mathrm{~N}$ ), low-amplitude fluctuations are detected,

524 which are seen as a zone of low gradient in panel (h). The model Laplacian in panel (i) illustrates

525 the resistive body in the south and the conductive zone in the north as downward and upward

526 curvature features, respectively.

527 Figure 6 shows a different cross-sectional view of the Krafla resistivity models; the view in

528 Figure 6 is perpendicular to the view in Figure 5 (see Figure 1 for exact orientation of these 
529 views). The vertical conductive structures seen at $7291 \mathrm{~N}$ in all three models in Figure 5(a), (d)

530 and (g) are clearly identifiable in all the resistivity sections in Figure 6(a), (d) and (g) centered at

$531417 \mathrm{E}$. All the structures are similar in width (2 km), but in the MT3Dinv model in panel (g), the

532 resistivity is lower ( 2 Ohm-m) and stops at $3.5 \mathrm{~km}$ depth. It is also evident that the resistive

533 bodies in the eastern part of the sections (422 E) vary considerably. The resistive body in panel

534 (a) is lower in amplitude (100-200 Ohm-m) and has a larger footprint (about $3 \mathrm{~km}$ wide)

535 compared to panel (d), where the body's width is $1.5 \mathrm{~km}$ between the $100 \mathrm{Ohm}-\mathrm{m}$ surface and the

536 peak amplitude of close to $800 \mathrm{Ohm}-\mathrm{m}$. The resistive body in panel (g) is highly resistive (>1000

537 Ohm-m) and forms a continuous feature from the surface to a depth of about $1 \mathrm{~km}$. The

538 conductive body ( $<10 \mathrm{Ohm}-\mathrm{m})$ on the eastern most end of the section (423 E) in panel (g) is not

539 seen in the other models; furthermore, this feature lies at the edge of the data coverage.

540 Therefore, it is likely that this model feature is an artifact of the inversion procedure. 

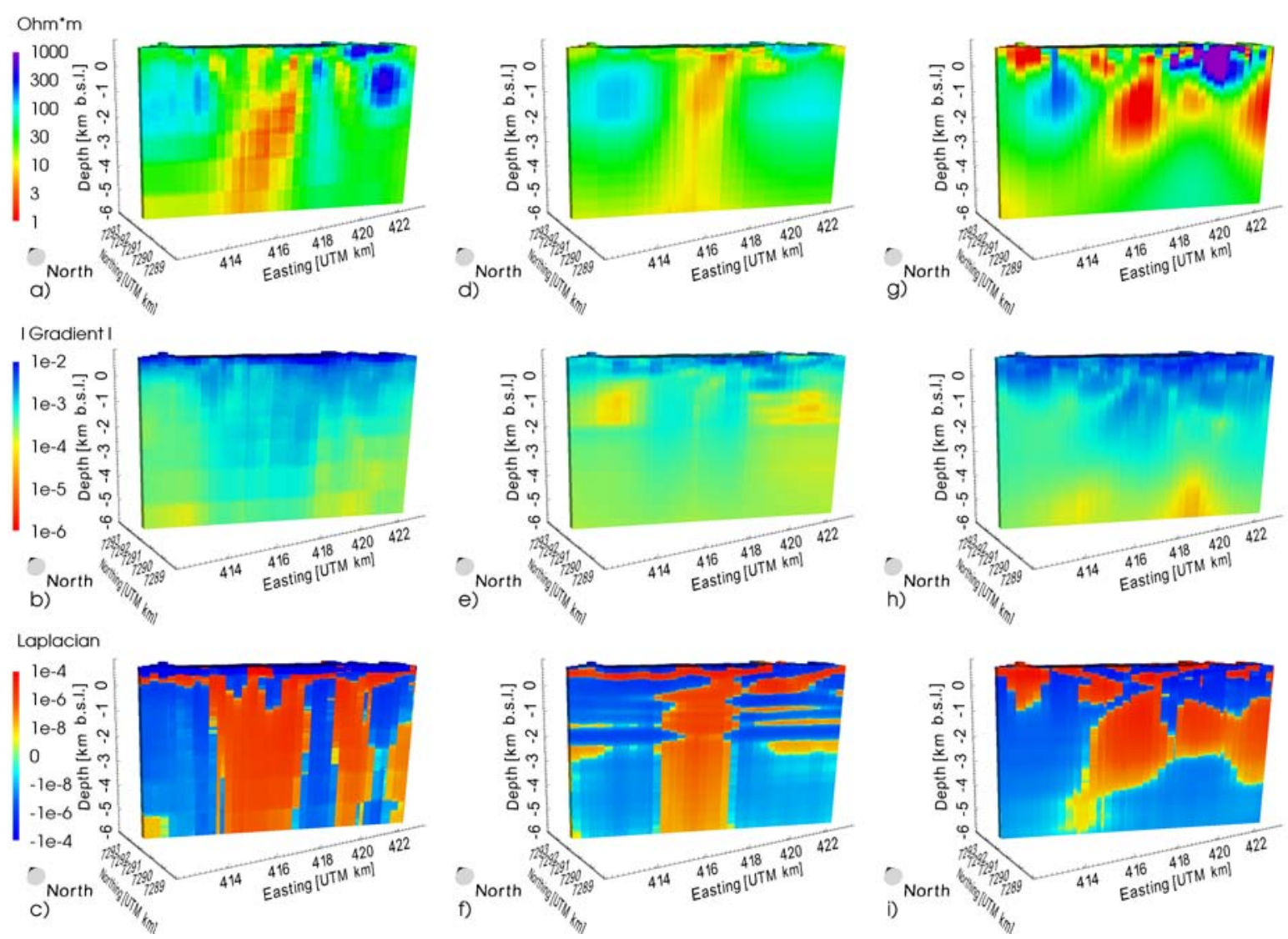

542 Figure 6. A cross section across the main fissure in Krafla (line B in Figure 1): (a), resistivity, (b) 543 gradient, and (c) Laplacian are for results obtained with WSINV3DMT; (d) resistivity, (e) 544 gradient, and (f) Laplacian are for results obtained with EMGeo; and (g) resistivity, (h) gradient, 545 and (i) Laplacian are for results obtained with MT3Dinv.

547 The gradients of the models in panels (b), (e) and (h), show that the changes are occurring at a

548 high rate (amplitude $>1 \mathrm{e}^{-4}$ ) in the near surface and at moderate depth in the central part of the 549 section, but below $4 \mathrm{~km}$ depth and closer to the edges of the models, gradient magnitude is close

550 to or below $1 \mathrm{e}^{-4}$. As in Figure 5, the Laplacian of the sections in Figure 6 illustrates the relative

551 structural coincidence of the vertical conducive bodies recovered in all the models. 
The vertical conductive structure reaching the near surface and the resistive core in the southwest

554 seen in the panels of Figure 5 and Figure 6 for all three models, increase confidence in the

555 geological importance of these structures. As well, all models have a conductive zone below a

556 surface resistor, but the shape and magnitudes of this conductor vary considerably between the

557 models. Comparison of the models at a depth of 1000 m.b.s.l. (Figure 7) reveals SW-NE and

558 NW-SE striking lineaments of structures in both the recovered models in panels (a) and (e).

559 However, no such features are noticeable in the recovered model in panel (c). Possible

560 explanations for these structural differences in the models are the variations in model

561 regularization and its weight in the objective function of the inversion codes.
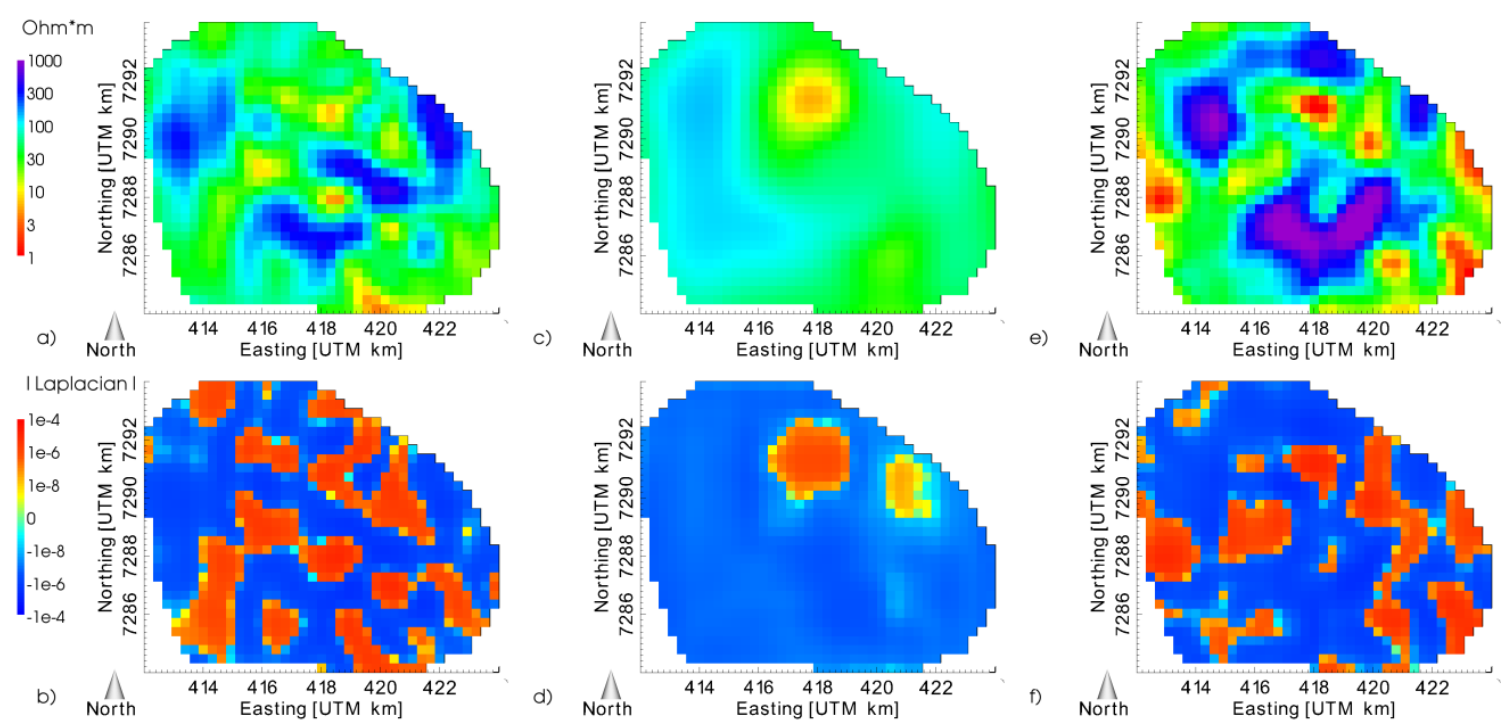

563 Figure 7. Depth slices through the Krafla models at depth of 1000 m.b.s.l. Panels (a) and (b) 564 show resistivity and Laplacian, respectively, for the results obtained with WSINV3DMT; (c) and 565 (d) show results obtained with EMGeo; and (e) and (f) show results obtained with MT3Dinv. In both panels (a) and (e) of Figure 7, the resistive structures in the northwest (414 E, 7290 N)

568 and south (418 E, 7288 N) are noticeable. The resistive features are broken up by linearly 569 trending conductive structures, striking SW-NE and NW-SE. The southeast resistive structure in 
570 panel (e) is seen as a single body with an isolated increased conductive feature in the center,

571 compared to a linear break in the same structure in panel (a). This is further seen in panels (b) and

572 (f) in the Laplacian of the models, where the linear aligned structures are emphasized. However,

573 the match is not complete: there are corresponding structures in both models, but the size and

574 shape differ considerably.

575 In comparing the model in Figure 7, panel (c), to the others in panels (a) and (e), we do not find

576 correspondence in the smaller scale structures. The structure in panel (c) has less detail: it shows

577 a large conductive feature in the northwest and a smaller feature in the southeast in an otherwise

578 fairly resistive background (higher than $80 \mathrm{Ohm}-\mathrm{m}$ ). The locations of these features correspond

579 to structures of the same dominant resistivity in panels (a) and (e), but the structures in panel (c)

580 have less detail. This is seen in panel (d), where only the conductive feature in the north is

581 rendered using curvature.

582 Figure 8 shows results using the cross-product comparisons described in Section 5.1 (section A in

583 Figure 1) where the normalized cross-gradient from EMGEO and MT3Dinv, WSINV3DMT and

584 EMGEO, and WSINV3DMT and MT3Dinv models are illustrated in panels (a), (b), and (c), 585 respectively. 

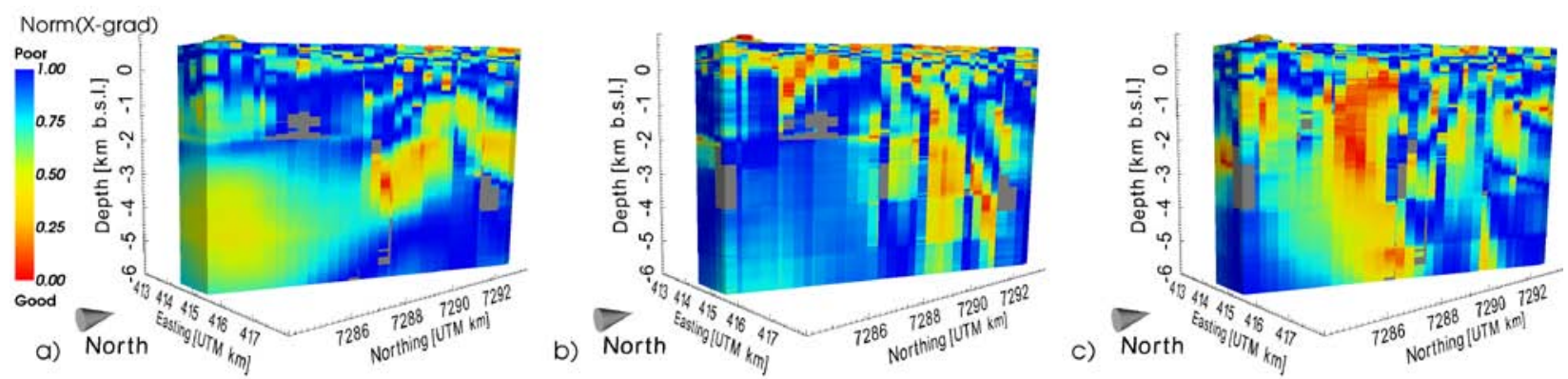

Figure 8. Normalized cross-gradient sections (section A in Figure 1) for the Krafla resistivity models: (a) EMGeo and MT3Dinv, (b) WSINV3DMT and EMGeo, and (c) WSINV3DMT and MT3Dinv, respectively. The grayed out area are zones where the gradient falls below threshold and cannot be reliably employed as described in Section 5.1. Values below 0.5 indicate good correlation between the respective models.

594 The cross-gradients shown in Figure 8 highlight the structural variation present in the models.

595 More structural correlation is found in the cross-gradients in panel (c), especially in the center of

596 the section associated with contact of the resistive body in the south. However, there appears to

597 be less structural correlation in other regions, especially at depth.

\subsection{Hengill}

599 The resulting inversion models using the inversion codes described in Sections 3.1, 3.2, and 3.3

600 are shown in Figure 9 and Figure 10 as cross-sections along and across the fissure directions in

601 the area, respectively (black lines A and B in Figure 3). All the models show the characteristic

602 structure of a high-temperature geothermal system: a resistive surface underlain by a conductive

603 zone, which resides over a resistive core region; and a deep conductor to the north. Also shown

604 are structural maps (gradient and Laplacian) for the models, where similarity in the maps indicate

605 structural correlations between respective models. 

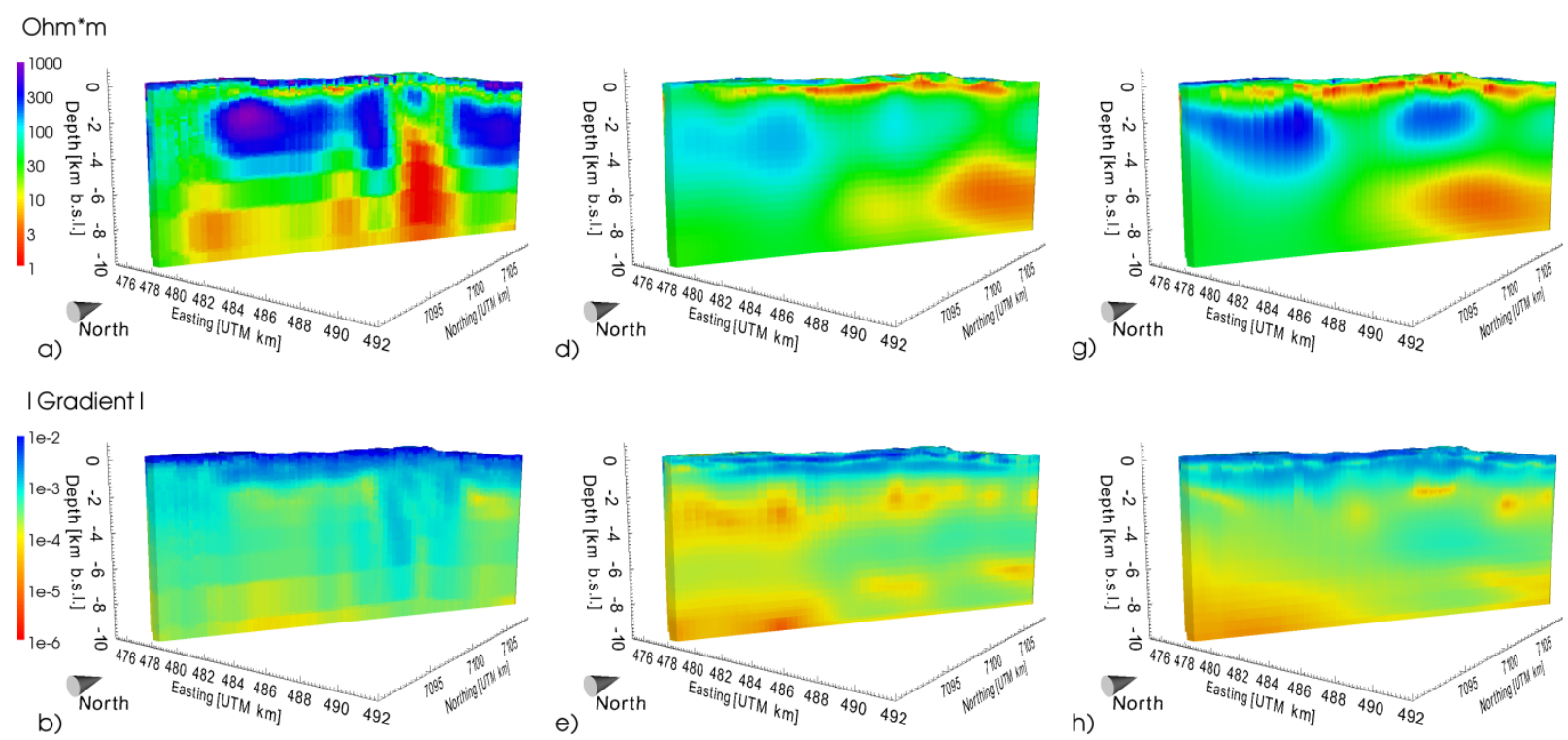

Laplacian
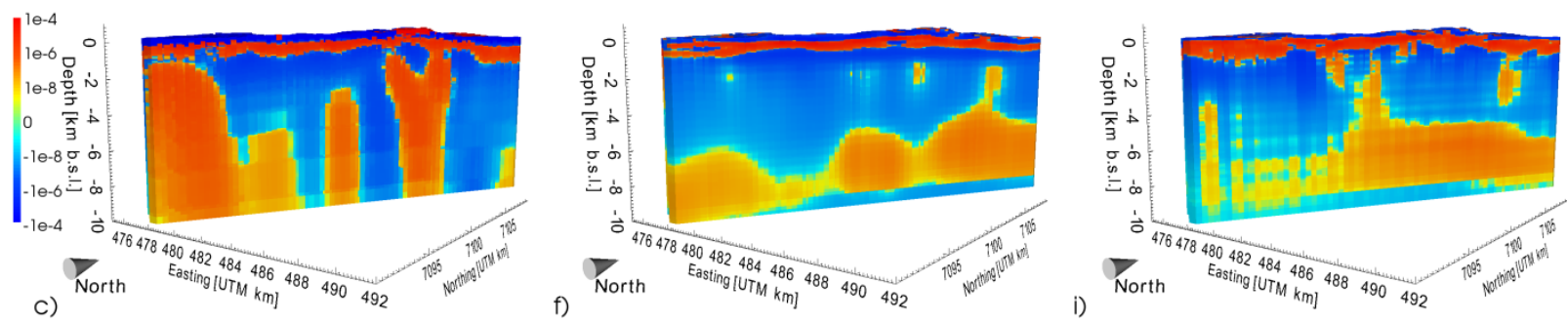

Figure 9. Cross-section along the main fissure swarm (line A in Figure 3) in Hengill showing (a) resistivity, (b) gradient, and (c) Laplacian for results obtained with WSINV3DMT; (d) resistivity,

609 (e) gradient, and (f) Laplacian for results obtained with EMGeo; and (g) resistivity, (h) gradient, 610 and (i) Laplacian, for results obtained with MT3Dinv.

612 The surface of the WSINV3DMT model in Figure 9 (a) is resistive $(\sim 500 \mathrm{Ohm}-\mathrm{m})$, with that

613 surface underlain by a thin conductive layer ( 10 Ohm-m). The resistive core is fairly uniform in

614 thickness, with resistivity between 500 to $1000 \mathrm{Ohm}-\mathrm{m}$, but is broken by a moderately

615 conductive zone $(\sim 50 \mathrm{Ohm}-\mathrm{m})$ in the southern end $(7090 \mathrm{~N})$ and a highly conductive zone $(<5$

616 Ohm-m) in the northern part of the section. There is considerable contrast in the resistivity

617 between the conductive breaks and resistive core (three orders of magnitude), which is

618 emphasized by the high-magnitude values of the gradient $\left(>1 \mathrm{e}^{-3}\right)$ in panel (b). Figure 9 (c) 
619 shows the Laplacian of the model, where the breaks in the resistive core are seen as pillars with 620 positive curvature.

621 The thickness of the conductive overburden in the EMGeo model shown in Figure 9 (d) is 622 noticeably greater than in panel (a). However, the resistive core in (d) is lower in amplitude 623 ( $\sim 150$ Ohm-m), and the deeper conductor is mainly observed in the northern part of the model.

624 Vertical conductive breaks ( $60 \mathrm{Ohm}-\mathrm{m})$ in the resistive core are observed in the center (482 E, $6257102 \mathrm{~N}$ ) and the northern part (484 E, $7105 \mathrm{~N}$ ) of the section. In panel (e), a relatively thick layer 626 (1-2 km thick) of low gradient (less than $\left.5 \mathrm{e}^{-5}\right)$ is mapped in the zone of the resistive core, 627 indicating a constant resistivity in the core. No clear changes in the gradient are observed 628 associated with the two conductive breaks in the resistive core, but the Laplacian in panel (f)

629 reveals a positive curvature in the northern more of the conductive breaks.

630 The MT3Dinv resistivity model in Figure 9 (g) shows very similar structures to the model in

631 panel (d). For example, a moderately resistive surface ( $200 \mathrm{Ohm}-\mathrm{m})$ is recovered, underlain by a 632 fairly continuous conductive layer that varies in thickness, as in the model in panel (d). The 633 resistive core is more resistive in panel (d) ( $500 \mathrm{Ohm}-\mathrm{m})$, with similar vertical conductive 634 breaks and at the same locations as in panel (d). The resistive core has a thin, low gradient layer $635\left(<5 \mathrm{e}^{-5}\right)$ and moderate lateral increase $\left(\sim 2 \mathrm{e}^{-4}\right)$, which coincides with the resistivity breaks in panel 636 (g). Both of these breaks have a positive curvature in the Laplacian in panel (i).

637 Figure 10 shows a different cross-sectional view of the Hengill resistivity models; the view is 638 close to perpendicular to the view in Figure 9 (see Figure 3 for exact orientation of these views).

639 As in Figure 9, the resistivity sections in panels (d) and (g) show considerable resemblance. A 640 resistive body is seen at the eastern end (490 E) in both sections, but a contrast in resistivity is 641 observable, where the model in panel (g) has a maximum of $600 \mathrm{Ohm}-\mathrm{m}$ compared to $200 \mathrm{Ohm}$ - 
$642 \mathrm{~m}$ in panel (d). Lower magnitude resistive body in the central part (485 E) is seen in both panels,

643 overlain by a noticeable thicker conductive layer. The model in panel (a) shares the character of

644 the other models in panels (d) and (g), but the near-surface conductive layer appears to be more

645 resistive (5 Ohm-m compared to 2 Ohm-m in models in panels (d) and (g) ) and the amplitude of

646 the resistive bodies are considerable greater. Examining the gradient in panels (e) and (h), reveals

647 the near-surface conductive layer to be a high magnitude gradient contrast, followed by the lows

648 associated with the resistive bodies. The gradient in panel (a) stands out as before, uncovering

649 vertical features around 490 E, which are supported by the Laplacian in panel (c), as contrasting

650 upward and downward curved features.
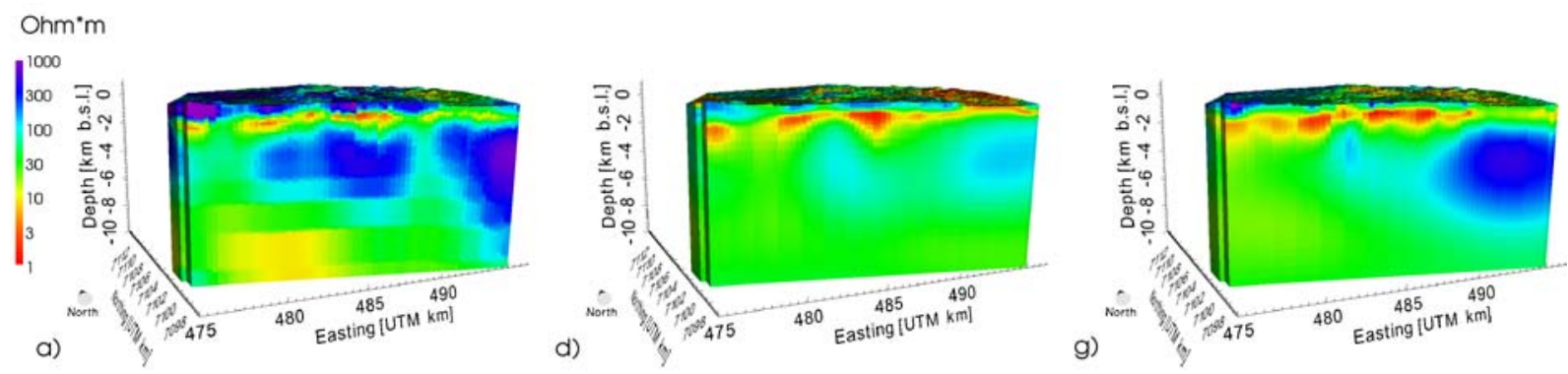

| Gradient I
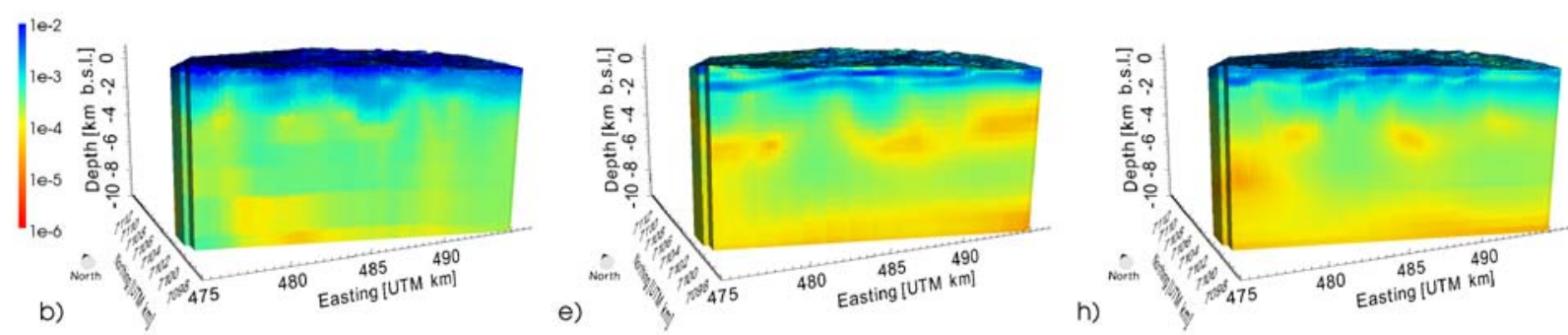

Laplacian
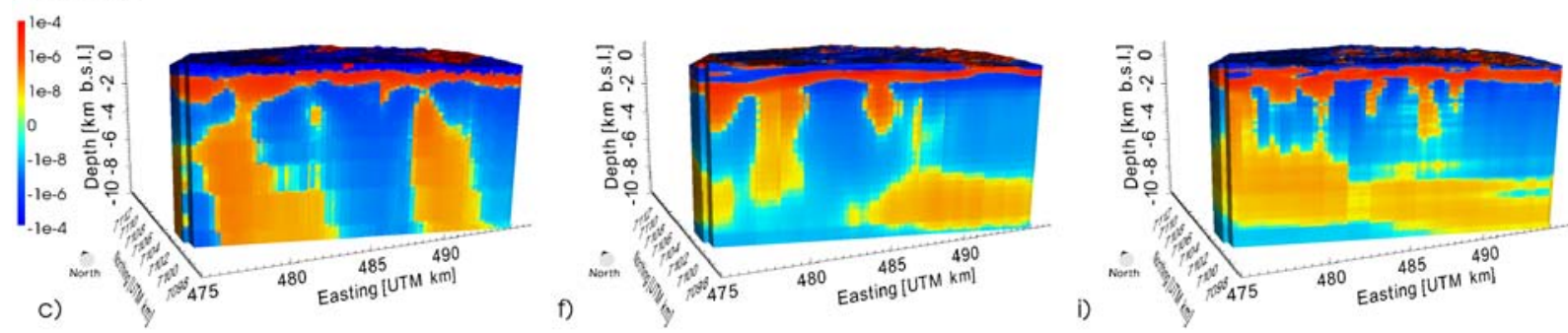

652 Figure 10. Cross-section across the main fissure swarm (line B in Figure 3) in Hengill showing 653 (a) resistivity, (b) gradient, and (c) Laplacian for results obtained with WSINV3DMT; (d) 
654 resistivity, (e) gradient, and (f) Laplacian for results obtained with EMGeo; and (g) resistivity, (h) 655 gradient, and (i) Laplacian, for results obtained with MT3Dinv.

657 By comparing the models and the structural derivatives in Figure 9 and Figure 10, one sees that

658 the similarities between the resistivity models in panels (d) and (g) are greater than for the model

659 in panel (a). However, all models agree on the main structures, recovering a resistive surface

660 layer with decreased resistivity coinciding with mapped surface alterations; a shallow conductive

661 layer that varies in depth and in thickness over the area; and a resistive core region that is broken

662 up by moderately conductive zones. The main differences in the WSINV3DMT model in panel

663 (a) compared with the other two models are the pronounced vertical structures, especially below

$6644 \mathrm{~km}$ depth. It is also noticeable that the cell resolution in the WSINV3DMT model is relatively

665 poorer at depth, due to the low number of vertical cells in the mesh.

666
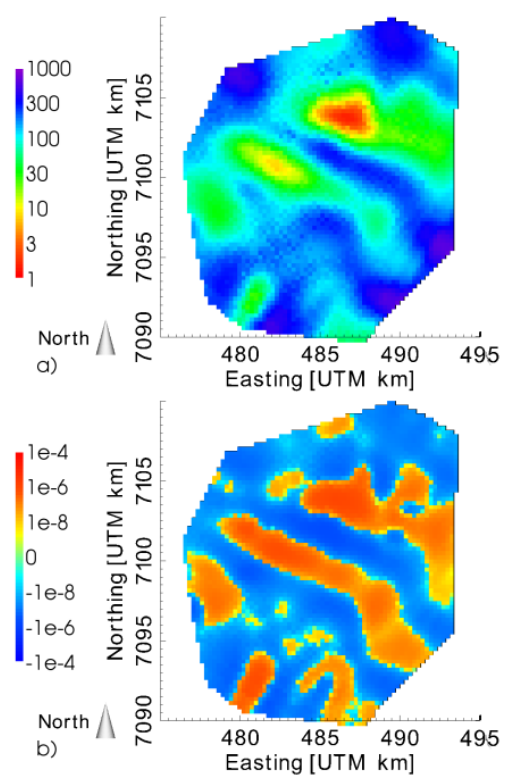

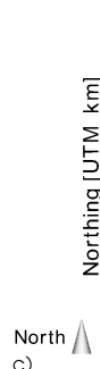

C)

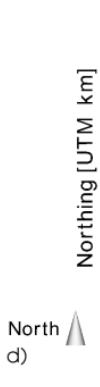

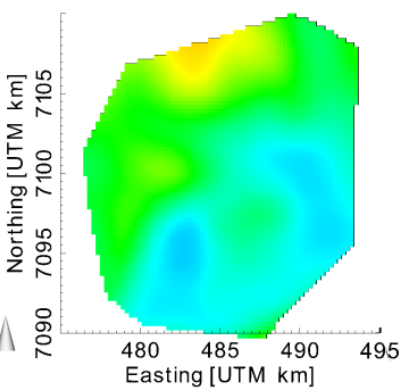

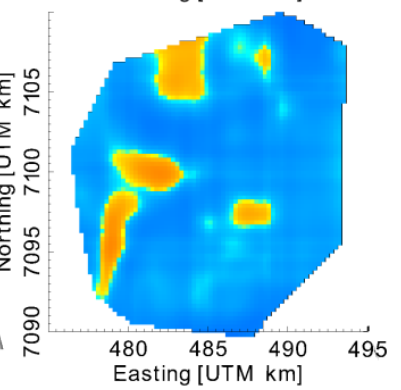

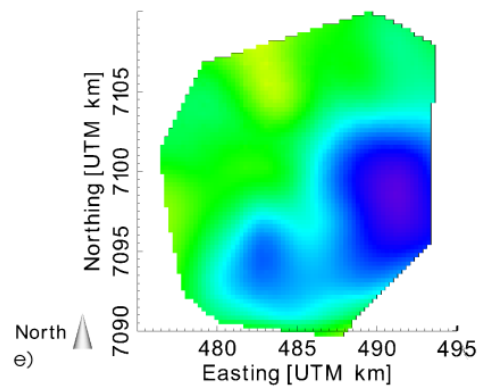

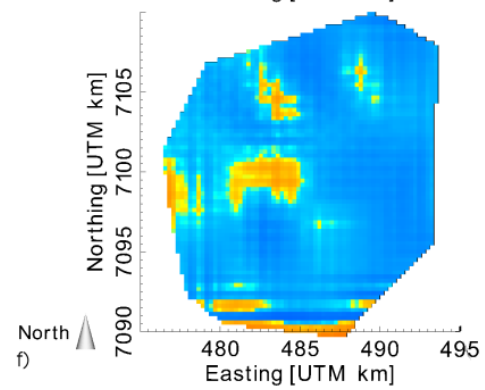

668 Figure 11. Depth slices through the Hengill models at depth of 4000 m.b.s.l. Panels (a) and (b) 669 show the resistivity model and Laplacian parameter, respectively, for results obtained with 
670 WSINV3DMT; (c) and (d) show corresponding results for EMGeo; and (e) and (f) show results 671 obtained with MT3Dinv.

673 Horizontal maps at a depth of 4000 m.b.s.l. are shown in Figure 11, where modeled resistivity

674 and Laplacian values are shown in panels (a) and (b), (c) and (d), and (e) and (f) for recovered

675 models from codes described in Section 3.1, 3.2, and 3.3, respectively. The structural

676 correspondence of the models in (c) and (e) is clear, with the southeast part of the sections

677 predominantly resistive (resistivity greater than 80 Ohm-m) and the northwestern part more

678 conductive. In panel (a), there are more permutations in the structure, with lineaments of

679 structures with a northwest-southeasterly strike present.

680 Figure 12 (a), (b), and (c) show the cross-gradient results from EMGEO and MT3Dinv,

681 WSINV3DMT and EMGEO, and WSINV3DMT and MT3Dinv, respectively.

682
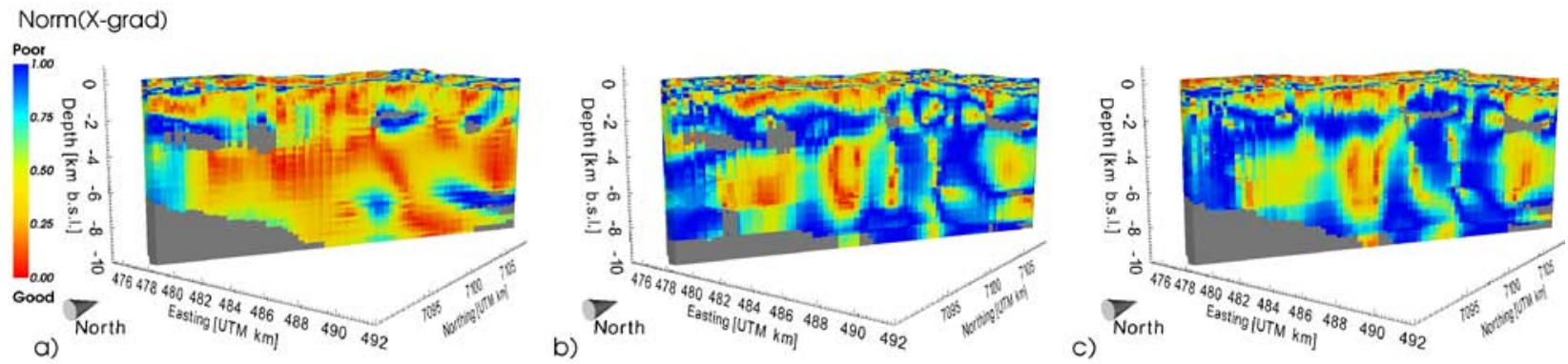

684 Figure 12. Normalized cross-gradient sections (line A in Figure 3?)for resulting resistivity 685 models at Hengill between (a) EMGeo and MT3Dinv codes, (b) WSINV3DMT and EMGeo 686 codes, and (c) WSINV3DMT and MT3Dinv codes, respectively. The grayed-out areas are zones where the gradient falls below threshold and cannot be reliably employed, as described in Section 5.1. Values below 0.5 indicate good correlation between the respective models.

690 The normalized cross-gradient presented in Figure 12 (a) confirms the similarities of the models

691 shown in Figure 9(b) and (c). Most of the values in Figure 12 (a) fall below 0.5, indicating a 
692 strong structural resemblance between the two models, confirming the observed similarities in

693 the resistivity models in Figure 9 and Figure 10. The main discrepancies are seen in the near

694 surface and at mid-depth in the resistive core region, where structural boundaries do not correlate

695 well. Panels (b) and (c) show the inconsistent interface at the top and bottom of near-surface

696 conductive layers in the models, seen as a thin layer of poor correlation (value close to 1). Major

697 discrepancies are seen at $486 \mathrm{E}, 7105 \mathrm{~N}$ in the section, where a conductive body is seen in the

698 model in Figure 9 (a) that is not recovered in the models in (d) and (g).

\section{6. Conclusions}

700 In this paper, we present a comparison of three 3D MT inversion codes using field data from the

701 Krafla and Hengill geothermal fields in Iceland. The goal was to assess the robustness of the

702 inversion procedure in a real data scenario, where results are known to strongly depend on the

703 quality of data, assignment of data uncertainties, model discretization, and other potential sources

704 of bias introduced during the inversion workflow. In general, the non-uniqueness of the 3D MT

705 inverse problem allows data to be fit to a similar level with different structures; confidence in a

706 particular model feature is thus derived from different modeling algorithms and settings

707 retrieving similar and coincident structures. The three inversion codes were run independently,

708 and it was up to the modeler to set up his/her inversion in an optimal way; the only restriction

709 was that all modelers had to use the same initial data sets.

710 Recovered resistivity values can be misleading where the dynamic range of recovered models can

711 be significantly different. Moreover, the differences between two models are not always obvious,

712 which can obscure interpretation. We show computing the model gradients, Laplacian, and

713 normalized cross-gradient can be useful, specifically when characterizing subtle structural 
714 features and for comparing two different models. These quantitative metrics are found to enhance

715 model appraisal and geological interpretation.

716 Different model results from Krafla are fairly inconsistent; however, all three codes recover:

717 (1) A fairly resistive surface, underlain by a conductive layer

718 (2) A deep conductive $(<10 \mathrm{Ohm}-\mathrm{m})$ zone to the north/northwest

719 (3) Resistive bodies in the south/southeast and northwestern parts of the survey area at

720 moderate depth.

721 Feature (2) rises from depths of $5 \mathrm{~km}$. Two inversion codes (WSINV3DMT and MT3Dinv)

722 recover models with more structure relative to the third code (EMGeo); nevertheless, structural

723 analysis reveals a fair degree of correspondence between all resistivity models. This structural

724 consistency, adds a level of confidence to the overall model interpretations, despite individual

725 model differences. One explanation for the large range of acceptable Krafla resistivity models is

726 data quality. Poor data quality can require the inversion codes to introduce extraneous structure in

727 an attempt to lower the data misfit.

728 The results from Hengill show more coincident resistivity structures relative to Krafla. All three

729 models recovered resistivity structures corresponding to the characteristic resistivity structure of

730 geothermal systems, with an up-doming around the central volcano. All models recover:

731 (1) A conductive (<10 Ohm-m) near-surface layer (within $1.5 \mathrm{~km}$ from the surface)

732 (2) A moderately deep resistive core ( $>100 \mathrm{Ohm}-\mathrm{m})$ broken in places by moderately

$733 \quad$ conductive zones

734 (3) A deep conductor in the northeastern part of Hengill. 
735 The greater consistency of recovered models at Hengill compared with the results from Krafla

736 increases confidence that these structures are required to fit the data, and are not artifacts from the

737 inversion process. Both EMGeo and MT3Dinv recover models that are structurally very similar,

738 as seen in the resistivity models, highlighted in the structural derivatives of the recovered models,

739 and further emphasized by cross-gradient analysis. The major difference between these two

740 models and WSINV3DMT model can be seen at $486 \mathrm{E}, 7105 \mathrm{~N}$, where the WSINV3DMT

741 recovers a vertical, relatively more conductive body compared to the moderately resistive zones

742 in the other two codes. One plausible explanation for this difference is inversion design; the mesh

743 used with WSINV3DMT expanded the thickness of cells much faster compared to EMGeo and

744 MT3Dinv; and in addition, both the grid and data were rotated by $30^{\circ} \mathrm{E}$.

745 The structural similarities of the resistivity models discussed in this paper increase confidence

746 that those structures are required by the data and arise from geological structures. Gasperikova et

747 al. (2015) (this issue) discuss these structures in greater detail, and in particular their relationship

748 to the known geology of the Krafla and Hengill geothermal areas.

749 7. Acknowledgements

750 This work was carried out at University of British Columbia and Iceland Geosurvey, with

751 funding provided by Geothermal Research Group GEORG; and at Lawrence Berkeley National

752 Laboratory, with funding provided by the Department of Energy Geothermal Program Office

753 under contract GT-480010-19823-10, and Office of Basic Energy Sciences under Award No. DE-

754 AC02-05CH11231. We also want thank three anonymous reviewers for the valuable comments

755 and suggestions to improve the manuscript. 


\section{References}

757

758

759

760

761

762

763

764

765

766

767

768

769

770

771

772

773

774

775

776

777

778

779

780

781

782

783

784

785

786

787

Árnason, K., Eysteinsson, H., Hersir, G.P., 2010. Joint 1D inversion of TEM and MT data and 3D inversion of MT data in the Hengill area, SW Iceland. Geothermics 39, 13-34.

Cagniard, L., 1953. Basic theory of the magneto-telluric method of geophysical prospecting. Geophysics 605-635.

Farquharson, C., Oldenburg, D., Haber, E., Shekhtman, R., 2002. An algorithm for the three-dimensional inversion of magnetotelluric data. 72nd SEG Annu. Meet. Salt Lake City, UT 649-652.

Flóvenz, Ó.G., Hersir, G.P., Sæmundsson, K., Ármannsson, H., Friðriksson, T., 2012. Geothermal Energy Exploration Techniques, in: Comprehensive Renewable Energy. Elsevier Ltd., pp. 51-94.

Friðleifsson, G.Ó., Ármannsson, H., Guðmundsson, Á., Árnason, K., Mortensen, a. K., Pálsson, B., Einarsson, G.M., 2014. Site selection for the well IDDP-1 at Krafla. Geothermics 49, 9-15.

Gamble, T.D., Goubau, W.M., Clarke, J., 1979. Error analysis for remote reference magnetotellurics. Geophysics 44, 959-968.

Gasperikova, E., Rosenkjaer, G.K., Arnason, K., Newman, G.A., Lindsey, N.J., 2014. Resistivity Characterization of The Kralfa and Hengill Geothermal Fielsds Through 3D MT Inverse Modelling. Geothermics Unpublsihe.

Heise, W., Caldwell, T.G., Bibby, H.M., Bannister, S.C., 2008. Three-dimensional modelling of magnetotelluric data from the Rotokawa geothermal field, Taupo Volcanic Zone, New Zealand. Geophys. J. Int. 173, 740-750.

Hjartardóttir, Á.R., Einarsson, P., Bramham, E., Wright, T.J., 2012. The Krafla fissure swarm, Iceland, and its formation by rifting events. Bull. Volcanol. 74, 2139-2153.

Kiyan, D., Jones, a. G., Vozar, J., 2013. The inability of magnetotelluric off-diagonal impedance tensor elements to sense oblique conductors in three-dimensional inversion. Geophys. J. Int.

Lindsey, N.J., Newman, G. a., 2015. Improved workflow for 3D inverse modeling of magnetotelluric data: Examples from five geothermal systems. Geothermics 53, 527-532.

Miensopust, M.P., Queralt, P., Jones, a. G., 2013. Magnetotelluric 3-D inversion--a review of two successful workshops on forward and inversion code testing and comparison. Geophys. J. Int. 193, 1216-1238.

Newman, G. a, Boggs, P.T., 2004. Solution accelerators for large-scale three-dimensional electromagnetic inverse problems. Inverse Probl. 20, S151-S170.

Newman, G. a., Alumbaugh, D.L., 2000. Three-dimensional magnetotelluric inversion using non-linear conjugate gradients. Geophys. J. Int. 140, 410-424. 
788

815

Newman, G. a., Gasperikova, E., Hoversten, G.M., Wannamaker, P.E., 2008. Three-dimensional magnetotelluric characterization of the Coso geothermal field. Geothermics 37, 369-399.

Oldenburg, D., Li, Y., 2005. Inversion for applied geophysics: a tutorial. Investig. Geophys. 1-85.

Oliphant, T.E., 2007. Python for Scientific Computing. Comput. Sci. Eng. 9.

Onacha, S., Shalev, E., Malin, P., Leary, P., Bookman, L., 2010. Interpreted Fracture Anomalies : Joint Imaging of Geophysical Signals from Fluid-Filled Fracture Zones in Geothermal Fields. Proc. World Geotherm. Congr. 2010 Bali, Indones. 25-29 April 2010 25-29.

Rosenkjaer, G.K., Oldenburg, D.W., 2012. 3D INVERSION OF MT DATA IN GEOTHERMAL EXPLORATION : A WORKFLOW AND APPLICATION TO HENGILL, ICELAND, in: ThirtySeventh Workshop on Geothermal Reservoir Engineering Stanford University, Stanford, California, January 30 - February 1, 2012. Stanford, California.

Rosenkjær, G.K., 2011. Electromagnetic methods in geothermal exploaration. 1D and 3D inversion of TEM and MT data from a synthetic geothermal area and the Hengill geothermal area, SW Iceland. University of Iceland.

Schroeder, W., Martin, K., Lorensen, B., 2006. The Visulization Toolkit: An Object-Oriented Approch To 3D Graphics, 4th Edition.

Siripunvaraporn, W., 2011. Three-Dimensional Magnetotelluric Inversion: An Introductory Guide for Developers and Users. Surv. Geophys. 33, 5-27.

Siripunvaraporn, W., Egbert, G., 2009. WSINV3DMT: Vertical magnetic field transfer function inversion and parallel implementation. Phys. Earth Planet. Inter. 173, 317-329.

Siripunvaraporn, W., Egbert, G., Lenbury, Y., Uyeshima, M., 2005. Three-dimensional magnetotelluric inversion: data-space method. Phys. Earth Planet. Inter. 150, 3-14.

Stark, M.A., Soyer, W., Hallinan, S., Watts, M.D., 2013. Distortion Effects on Magnetotelluric Sounding Data Investigated by 3D Modeling of High-Resolution Topography. GRC Trans. 37.

Vozoff, K., 1991. The magnetotelluric method, in: Naibighian, M. (Ed.), In: Naibighian M (ed) Electromagnetic Methods in Applied Geophysics. Society of Exploration Geophysicists, Tulsa, Oklahoma, pp. 641-711. 
817 Appendix A.

818 In the section we discuss and give examples of the parameters defined in Section 5.1.1. Since this

819 analysis uses $\log 10$ of the resistivity values, a change from 1000 to $100 \mathrm{Ohm}-\mathrm{m}$ is equivalent to a

820 change from 10 to $1 \mathrm{Ohm}-\mathrm{m}$ between adjacent cells. Thus, if the distance between these cell

821 centers were $100 \mathrm{~m}$, the resulting gradient magnitude is $10^{-2}$. The Laplacian is sensitive to the

822 sign of the gradient, resulting in either a positive or negative value depending on whether

823 resistivity is increasing or decreasing, respectively. One cannot determine whether resistivity is

824 increasing or decreasing solely by the gradient. 


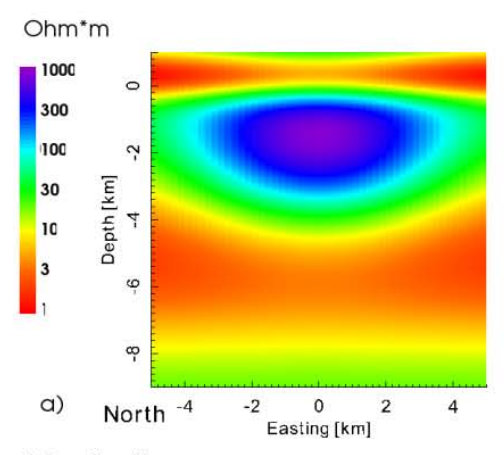

d)

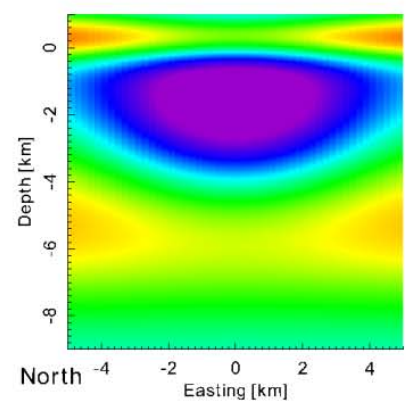

e)
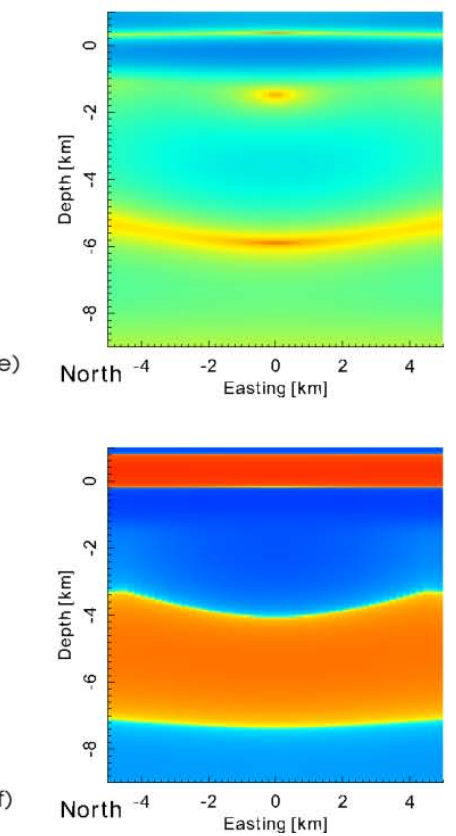
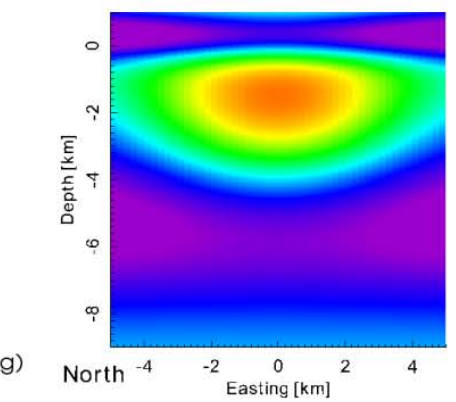

h)
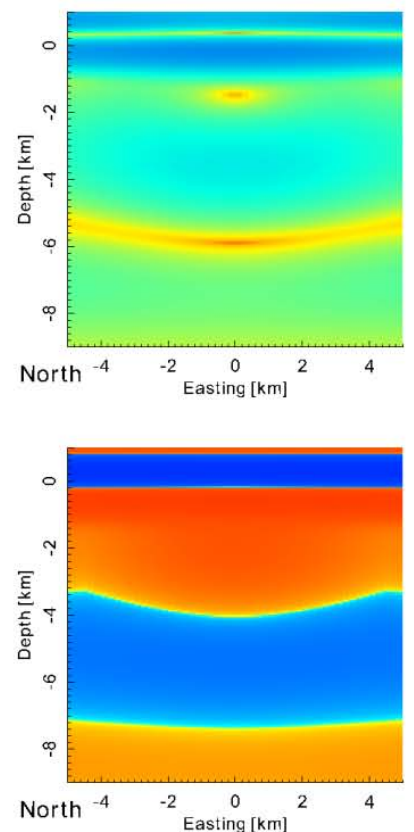

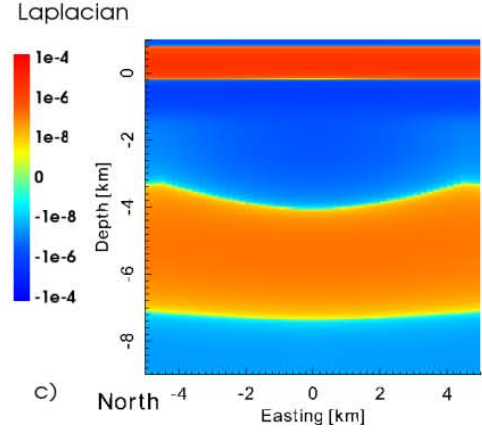

826 Figure 13. Synthetic models with simple resistivity structures are shown in (a), (d), and (g), with the corresponding magnitude of the gradient and Laplacian shown in (b) and (c); (e) and (f); (h) and (i), respectively. Relative changes in the structural trends in all the models are the same, seen as identical gradients in for all the models (b), (e), and (h). Notice that when a conductive body replaces a resistive body (as in (g), contrasting with (a) and (d)), this change is reflected in the

831 Laplacian measure but not in the magnitude of the gradient.

An illustration of these parameters is shown in Figure 13. Simple resistivity models are shown in

834 the top row (panels a, d, g). All the models can be characterized as three structures added to a

835 background resistivity value; only the background and the relative amplitudes of the embedded

836 structures change (i.e., the structural geometry is unchanged). Model (a) has a relatively 
838 backgrounds of 100 Ohm-m. Despite this difference, the gradient of the models, shown in the

839 middle row (panels b, e, h), ) are the same, demonstrating that the background resistivity value has

840 no effect on the gradient. The structures in Models (a), and (d) were constructed by adding

841 positive resistivity values to the background, while the structure in Model (g) was constructed by

842 adding negative resistivity values to the background. The opposite amplitude of the structures is

843 not detected in the magnitude of the gradient vector. However, the Laplacian of the models,

844 shown in the bottom row (panels c, f, i), does show structural trends. For example, the Laplacians

845 of model (a) and (d) shown in panels in (c) and (f), respectively, clearly show the models

846 exhibiting similar structural trends, while the Laplacian of the model in (g) shown in panel (h), is

847 clearly different. Since the gradients of all the models are the same, the cross-gradient is zero for

848 all of them.

849

850 


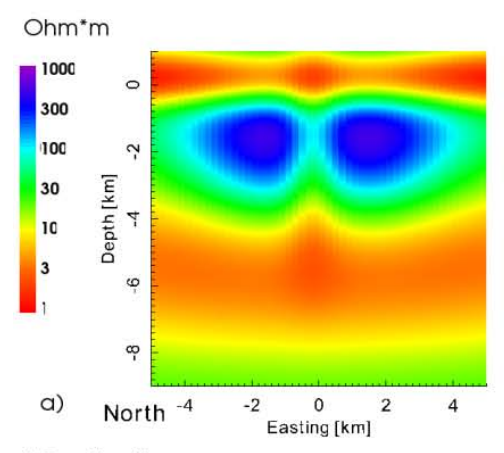

d)

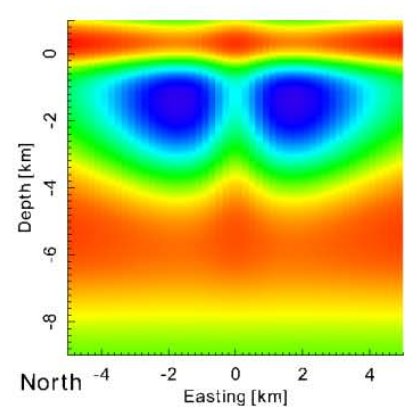

e)
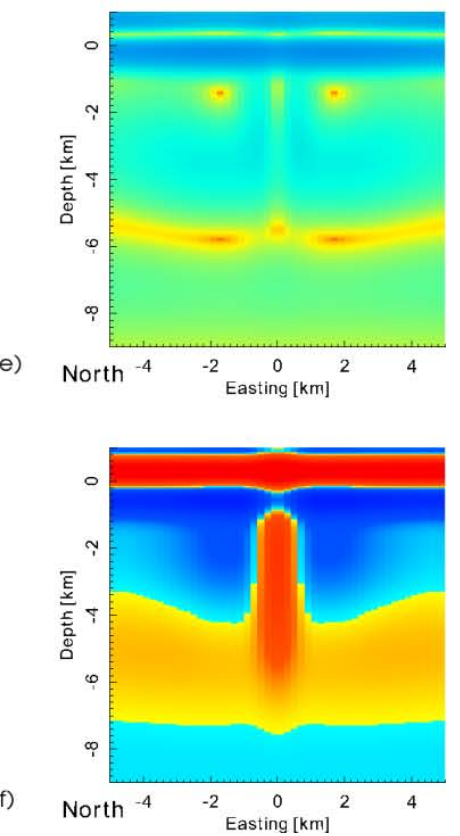
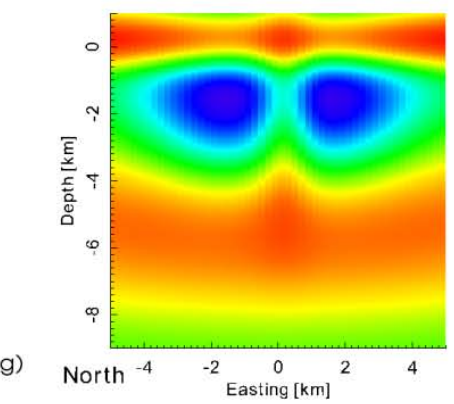

h)
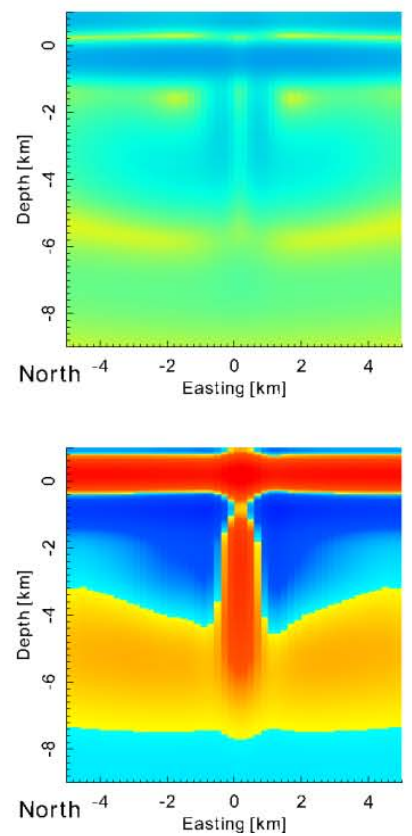

Laplacian

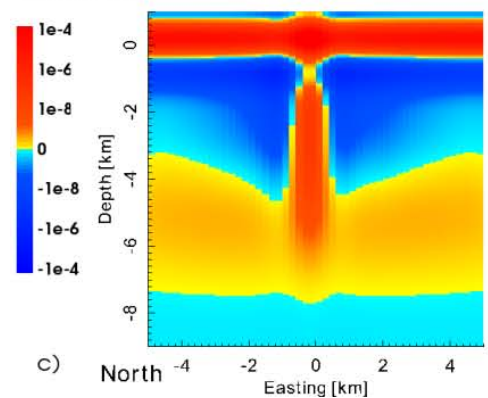

852 Figure 14. Synthetic models with simple resistivity structures are shown in (a), (d), and (g), with the corresponding magnitude of the gradient and Laplacian shown in (b) and (c); (e) and (f); (h) and (i), respectively. The models all have the same background resistivity and same structures, but amplitude peaks are shifted in space, which are not easily detected without recourse to structural metrics, such as the gradient and Laplacian discussed in this paper.

Figure 14 shows cross-sectional views of three resistivity models that all have the same

859 background resistivity of 30 Ohm-m. Each model is embedded with similar structures, a shallow

860 conductive layer, a split resistive body, and a deep conductive layer. The structural dissimilarities

861 of the models arise from spatial shifting of the amplitude peaks in the shallow and deep 
centered, but in Models (a) and (g), the structures are shifted symmetrically about the center by

864300 m, 800 m, and 2000 m laterally. The visual similarity of Models (a), (d), and (g) is clear.

865 Panels (b), (e), and (h) show how these models have similar gradient magnitudes, but some

866 differences can be seen. For example, in panel (e), the resistive structures and the deep

867 conductive layer produce relatively large gradients where magnitudes below $1 \mathrm{e}^{-5}$ are reached.

868 Likewise, the Laplacian of Models (a), (d), and (g), shown in panels (c), (f), and (i), exemplify

869 subtle differences. Nonetheless, the Laplacian for all the models shows a positive curvature

870 associated with the break in the resistive layer.

$\operatorname{Norm}(X-g r a d)$

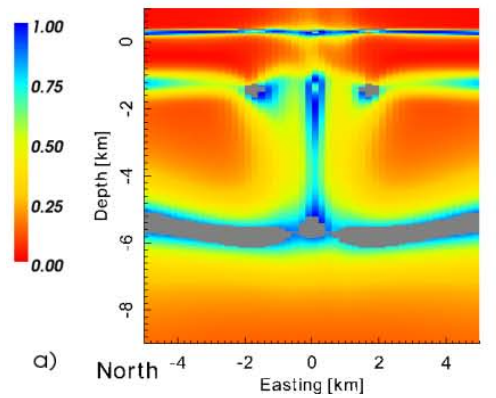

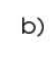

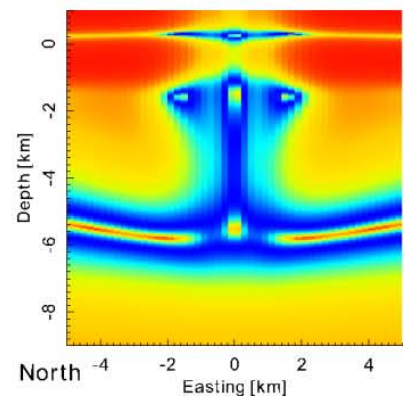

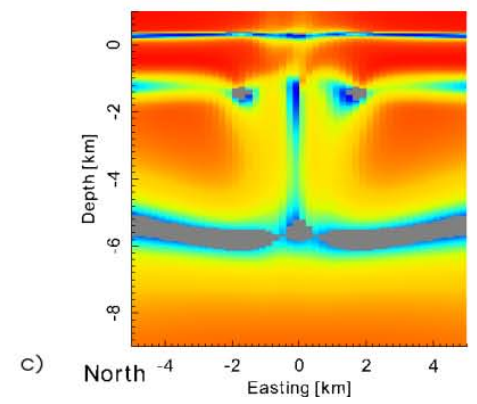

872 Figure 15. Cross-gradients of resistivity models shown in Figure 14: (a) the cross-gradient of 873 models in Figure 14 (d) and (g), (b) cross-gradient of models in Figure 14 (a) and (g), and (c) 874 cross-gradient of models in Figure 14 (a) and (d). The gray regions in these plots are areas where 875 either one of the gradients is very small, making the value of the cross-gradient NaN, and thus no 876 color is plotted (see Section 5.1 for further details).

Figure 15shows the cross-gradients of the resistivity models in Figure 14 (a), (d), and (g). While

879 the parameters shown in Figure 14 reveal structural similarities between models, the cross-

880 gradient parameter highlights model differences. The cross-gradients in panels (a) and (c) look

881 similar, illustrating the symmetric spatial shifts of the structures shown in Figure 14(a) and (g),

882 compared to the structure in (d). The grayed-out area in both figures corresponds to the low

883 magnitude in the gradient in Figure 14(e), discussed above, which makes the cross-gradient 
884 difficult to determine (see Section 5.1 for further details). The cross-gradient in panel (b) exposes 885 dissimilarities in gradient structures associated with the break in the resistive and the deep

886 conductive layers. The magnitude of the normalized cross-gradient is high in these regions,

887 indicating that the angle between the gradient vectors is approaching $90^{\circ}$.

888 The idealized modeling examples discussed above highlight advantages and disadvantages of the 889 proposed metric parameters with respect to mapping structural coincidence in the models. Using

890 different structural metrics together enables interpretation of subtle resistivity model features, and 891 enhances comparison and model appraisal. 